\title{
Astaxanthin Protects Against Early Acute Kidney Injury in Severely-Burned Rats Through Inactivating TLR4/MyD88/NF-kB Axis and Upregulating Heme Oxygenase-1
}

\section{Songxue Guo}

The Second Affiliated Hospital Zhejiang University School of Medicine

\section{Linsen Guo}

Changzhou No.7 People's Hospital

\section{Quan Fang}

The Second Affiliated Hospital Zhejiang University School of Medicine

\section{Meirong Yu}

The Second Affiliated Hospital Zhejiang University School of Medicine

\section{Liping Zhang}

The Second Affiliated Hospital Zhejiang University School of Medicine

\section{Chuangang You}

The Second Affiliated Hospital Zhejiang University School of Medicine

\section{Xingang Wang}

The Second Affiliated Hospital Zhejiang University School of Medicine

Chunmao Han ( $\nabla$ zrssk@zju.edu.cn)

Zhejiang University School of Medicine Second Affiliated Hospital https://orcid.org/0000-0002-28211323

\section{Research}

Keywords: astaxanthin, acute kidney injury, burn, inflammation, toll-like receptor 4, heme oxygenase-1

Posted Date: October 7th, 2020

DOl: https://doi.org/10.21203/rs.3.rs-84446/v1

License: (1) This work is licensed under a Creative Commons Attribution 4.0 International License. Read Full License 


\section{Abstract}

Background: Early acute kidney injury (AKI) contributes to severe morbidity and mortality in criticallyburned patients. Renal inflammation plays a vital role in the progression of early AKI. Astaxanthin (ATX) is a strong antioxidant widely-distributed in marine organisms, exhibiting diverse biological effects in trauma and diseases. The anti-inflammatory property of ATX is also suggested. Hence, we attempted to explore the anti-inflammation-based protection of ATX against early AKI post-burn and related mechanisms.

Methods: A severely-burned rat model was established in this study. The changes of renal structure and function were determined by hematoxylin-eosin staining and blood test. The oxidative status in kidneys was detected by commercial kits and quantitative real time PCR. Furthermore, immunofluorescence staining, quantitative real-time, and western blot were performed on renal tissues of burned rats to explore the underlying effects and mechanisms of ATX on burn-induced AKI with the help of inflammation inducer and several signal inhibitors.

Results: We found oxidative stress-induced tissue inflammation participated in the development of early $\mathrm{AKI}$ after burn paralleling with the deterioration of histological damage and function in kidneys, and MyD88-dependent TLR4/NF-kB pathway was activated to regulate renal inflammation. TLR4 and NF-kB inhibitor-TAK242 and PDTC showed similar effects to attenuate burn-induced renal inflammation and early AKI. Upon ATX treatment, the release of inflammatory mediators in kidneys was downregulated, while TLR4/MyD88/NF-kB axis was inhibited dose-relatedly. Lipopolysaccharide (LPS) could reverse the anti-inflammatory effect of high-dose ATX, whereas TAK242 and PDTC antagonized its action. Furthermore, the expression of heme oxygenase $(\mathrm{HO})-1$ was also dose-relatedly upregulated by ATX.

Conclusions: Collectively, the data above suggests ATX dose-relatedly protects against renal inflammation through regulating TLR4/MyD88/NF-kB axis and HO-1, and finally prevent early AKI following severe burns.

\section{Background}

Severely burned patients (total body surface area (TBSA) $\geq 20 \%$ ), have to face a series of devastating circular or organ issues after initial thermal insult, which could be largely attributed to burn-induced ischemia/hypo-reperfusion, oxidative damage, and secondary inflammation or cell death ${ }^{1-3}$. As an organ with rich blood supply and poly-unsaturated fatty acid, kidney is vulnerable to attack from reactive oxygen species (ROS) and following inflammatory response under an ischemic condition and develops subsequent $A K I^{4,5}$. In terms of burn injury, early acute kidney injury (AKI) usually occurs within the first 3 days after trauma and contributes to a poor prognosis and even a high mortality (80\%) and morbidity, which is apt to give rise to a series of rapid pathologic damages on tubular and glomerular structures and predisposes, presenting a sequentially functional deterioration of kidney ${ }^{2,6}$. The incidence of acute renal dysfunction in burn patients receiving critical care can be up to $40 \%^{2}$. We have previously provided 
several evidences to support the key roles of ROS-related oxidative stress and secondary local mitochondria-related apoptosis in pathophysiological issues during the progression of burn-induced early $\mathrm{AKI}^{7}$. Furthermore, we also found some clues to take renal tissue inflammation into account as another important issue following ischemia or ROS burst ${ }^{8}$. Multiple studies, referring to severe trauma (including burn), large surgery and sepsis, have suggested the effectiveness of anti-inflammatory strategy to protect against AKI or other acute organ injuries following above conditions ${ }^{8-10}$. However, the way of early and timely interventions to burn-induced organ inflammation is still worthy to be further explored.

Nuclear factor(NF)-KB is regarded as a potential and crucial intermediate regulator for local inflammatory condition in early AKI after burn ${ }^{8}$. We also observed the selective antioxidant-molecular hydrogen may be able to inhibit burn-induced release of inflammatory cytokines in rat kidneys through a NF-KB-involved signaling pathway, and finally attenuate early AKI accompanying with its effect on apoptosis ${ }^{8}$. Futhermore, toll-like receptor (TLR)4/NF-kB signaling pathway has been suggested to be a classic cascade, which is able to regulate the development of inflammatory response in diverse organ injuries following ischemia-reperfusion (IR) ${ }^{11}$. Moreover, the effects of nephroprotectants and precondition treatment on different kinds of kidney injury are also closely related to the downregulation of signals included in TLR4/NF-KB pathway ${ }^{5,12}$. Recently, several researchers observe the regulatory role of TLR4 pathway in burn-induced sepsis, lung or cerebral injury, as well as a hopeful therapeutic target. In addition to inflammation, reactive oxygen species (ROS) burst also accompanies with the expression some endogenous antioxidant proteins, like heme oxygenase-1(HO-1), Nrf2, etc., in some ischemic or LPSinduced organ injuries, as a self-defense response ${ }^{13,14}$. HO-1 is reported a nephro-protective role in renal injury caused by diverse stimuli, due to its antagonism against oxidative damage and its regulation on several biological progress ${ }^{13,15-18}$. Considering burn-caused hypodynamic circulation and increased oxidative stress, TLR4/NF-KB pathway and HO-1 seems to be potential players in the development of burn-induced early AKI.

Astaxanthin (3,3'-dihydroxy-b, b'-carotene-4,4'-dione, ATX), as a natural carotenoid easily obtained from marine organisms, exhibits a more robust and powerful anti-oxidative effects than other carotenoids ${ }^{19}$. Previously, researchers has demonstrated that pretreatment or immediate administration of ATX is able to attenuate oxidative stress induced toxicity in tubular epithelial cells and I/R induced or diabetes-related renal injury in mice via reducing oxidative stress, inflammation and tubular apoptosis ${ }^{20-22}$. In terms of burn injury, we observed that ATX showed a protective effect of burn-induced early AKI by ameliorating oxidative damage and downregulating mitochondria-related apoptosis via PI3K/Akt/Bad pathway ${ }^{7}$. Combined with similar reports of its effects on oxidative damage and inflammation in other organ injuries, ATX seems to be a hopeful and non-toxic therapeutic reagent to prevent early AKI after burn ${ }^{23-25}$.

Given the important roles of oxidative and secondary renal inflammation in severe burn-induced early AKI, we hypothesized the possible anti-inflammation-based protection and potential mechanisms of ATX action on early AKI after burn through regulating TLR4/NF-KB pathway and HO-1, and aimed to explore more details to support clinical treatment. 


\section{Materials And Methods}

\section{Experimental animals and severe burn model establishment}

The current study was performed according to protocols approved by the Committee of The Second Affiliated Hospital Zhejiang University School of Medicine on Animal Care and Use (2016-144), and strictly followed the National Institutes of Health Guidelines for the Care and Use of Laboratory Animals. Adult male Sprague-Dawley (SD) rats, weighing approximately 220-250 g, were purchased from the Shanghai Slac Laboratory Animal Company (Shanghai, China) and housed with a 12-hour light/dark cycle in a filtered-air unit at a constant temperature and humidity. Additionally, all animals were free access to food and water.

The severely-burned rat model was established in accordance with previous reports ${ }^{7,8}$. Specially, after intraperitoneally injecting (ip) sodium pentobarbital $(50 \mathrm{mg} / \mathrm{kg})$, the model was produced by exposing the rats to a $15 \mathrm{~s}$ immersion into $100{ }^{\circ} \mathrm{C}$ hot water to generate a large-scale full-thickness dermal burn. The thermally-damaged area occupied approximately $40 \%$ of TBSA. The rats in the sham group were treated by $25^{\circ} \mathrm{C}$ water on the shaved dorsum after anaesthesia. During operation, the breath and heart rate of burn rats were carefully monitored to ensure all rats were under anesthetic and painless before postanesthesia recovery. Liquid resuscitation was proceeded by intraperitoneally injecting lactated Ringer solution (LRS) at $4 \mathrm{~mL} / \mathrm{kg} /$ TBSA immediately and $6 \mathrm{~h}$ after the operation. In addition, all of rat models were housed in individual cages and administered $0.25 \mathrm{mg} / \mathrm{kg}$ of buprenorphine by subcutaneous injection immediately and every $12 \mathrm{~h}$ post burn for analgesia. A pain and distress scale reported previously was introduced to instruct pain-reliving therapy ${ }^{7}$.

\section{Animal grouping and Drugs administration}

The whole study was divided into two parts to explore different purposes. In the part I, Eighty-four SD-rats were randomly assigned to eight groups, including the sham group, the four burn groups $(6 \mathrm{~h}, 12 \mathrm{~h}, 24 \mathrm{~h}$, $48 \mathrm{~h}$ ), the vehicle treatment (burn plus vehicle1) groups, the burn plus TAK242 or PDTC groups $(\mathrm{n}=8$ per group). Both TAK242 (MCE, USA) and PDTC (Beyotime, China) were dissolved in a vehicle solution (Vehicle1, 1\% dimethyl sulfoxide in distilled water). Animals in TAK242 or PDTC group were respectively given TAK242 at a dosage of $3 \mathrm{mg} / \mathrm{kg}$ or PDTC at a dosage of $100 \mathrm{mg} / \mathrm{kg}$ by tail-intravenous or ip injection $0.5 \mathrm{~h}$ pre-burn ${ }^{26-29}$. Both sham and burn groups received equal volumes of distilled water, while the burn plus vehicle1 group was administrated with equal volume of vehicle1 solution.

In the part II, Eighty SD-rats were randomly assigned to ten groups, including the sham group, the LPS treatment group, the three ATX-treatment groups, the ATX plus LPS group, and the ATX plus LPS and TAK242 or PDTC groups ( $\mathrm{n}=8$ per group). ATX (Sigma-Aldrich, St. Louis, MO, USA) solution was prepared in Vehicle2 (polyethylene glycol 400-N,N-dimethylacetamide (PEG400) purchased from Sigma-Aldrich, St. Louis, MO, USA (50:50, v/v), ), at concentration of $5 \mathrm{mg} / \mathrm{ml}, 10 \mathrm{mg} / \mathrm{ml}$ and $20 \mathrm{mg} / \mathrm{ml}$. The rats in ATXtreatment groups respectively received ATX at $5 \mathrm{mg} / \mathrm{kg}, 10 \mathrm{mg} / \mathrm{kg}$ and $20 \mathrm{mg} / \mathrm{ml}$ by tail iv injection at different doses ${ }^{7,30}$. In the sham group, rats were given equal volumes $(1 \mathrm{ml} / \mathrm{kg})$ of distilled water by tail iv 
injection. The animals in vehicle2 groups were given $1 \mathrm{ml} / \mathrm{kg}$ of PEG400 (50:50, v/v) without any drugs by tail iv injection. All above treatments above were administrated at $30 \mathrm{~min}$ after surgery ${ }^{7,30}$. The LPS control group received $4 \mathrm{mg} / \mathrm{kg}$ Lipopolysaccharide (LPS) immediately after sham operation by ip routine $^{31}$. The ATX plus LPS group was given $20 \mathrm{mg} / \mathrm{kg}$ ATX 30 min post-burn and $4 \mathrm{mg} / \mathrm{kg}$ LPS $1 \mathrm{~h}$ after burn induction by ip injection ${ }^{31,32}$. The TAK242 and PDTC were tail-intravenously or intraperitoneally administrated $0.5 \mathrm{~h}$ pre-burn at $3 \mathrm{mg} / \mathrm{kg}$ and $100 \mathrm{mg} / \mathrm{kg}$ in the selected ATX plus LPS groups as mentioned above.

\section{Histological preparation}

The rats in all groups were sacrificed by overdoses of sodium pentobarbital. Except those in the three burn groups (sacrificed at 6 h, 12 h, 24 h post burn), the animals in the other groups were sacrificed at 48 $\mathrm{h}$ post insults. Both kidneys were dissected after cardiac perfusion with phosphate-buffered saline (PBS) $(\mathrm{pH}=7.2)$ and were maintained in $4 \%$ paraformaldehyde at $4^{\circ} \mathrm{C}$ or in a $-80^{\circ} \mathrm{C}$ freezer for subsequent assessment. The frozen or paraffin-embedded kidney samples were sectioned by using a Cryostat Microtome or a Rotary Microtome (Leica, Solms, Germany) for further staining.

\section{Histological examination}

Hematoxylin and eosin (HE) staining was performed for histological examination, and the stained slices were observed and snapped under a microscope (DM2500, Leica, Solms, Germany). Twelve high magnification files were randomly selected for observation from six slices per group in a blind manner from two pathologists. The tubular damage score was determined based on the percentage of injurious renal cortical tubules and ranked as 0: normal, 1: less than $10 \%, 2: 11$ to $25 \%, 3: 26$ to $75 \%$, and 4: greater than $75 \%$.

\section{Renal function evaluation}

Blood samples were collected to measure levels of serum creatinine $(\mathrm{s} C r)$ via a clinical chemistry analyzer system and kits (Au5800, Beckman coulter, CA, USA). The serum Neutrophil gelatinaseassociated lipocalin (NGAL) levels were detected by using a Rat NGAL ELISA kit (EK0855, Boster, Wuhan, China) according to the manufacturer's instructions.

\section{Immunofluorescence staining}

The 7- $\mu \mathrm{m}$-thick frozen slices were rewarmed, washed in PBS for $10 \mathrm{~min}$, and then received the antigen retrieval. After incubation with hydrogen peroxide for $10 \mathrm{~min}, 5 \%$ bovine serum albumin (BSA) was applied as the blocking solution for $20 \mathrm{~min}$ at room temperature. Without washing, the sections were incubated with anti-TLR4 (1:100, ab22048, Abcam, Cambrige, UK) or anti-HO-1 (1:200, ab13243, Abcam, Cambrige, UK) antibody overnight at $4{ }^{\circ} \mathrm{C}$. Rinsed with PBS, the sections were incubated respectively with a FITC (1:50, BA1101, Boster, Wuhan, China) labeled goat anti-mouse or Cy3 (1:50, BA1032, Boster, Wuhan, China) labelled goat anti-rabbit secondary antibody for $2 \mathrm{~h}$ at $37^{\circ} \mathrm{C}$ in the dark. The sections were 
rinsed and stained with DAPI ( $100 \mathrm{ng} / \mathrm{ml}$; Boster, Wuhan, China) for $8 \mathrm{~min}$ at room temperature and then mounted with Vectashield mounting medium (H-1000, Vector, CA, USA). All of the slices were observed and snapped under a fluorescent microscope (DM5500B, Leica, Solms, Germany).

\section{Immunohistochemistry (IHC) staining}

IHC staining was performed for evaluation on distribution of inflammation mediators in the renal tissues. After deparaffinization, rehydration and antigen retrieval, the sections were incubated with anti-MPO antibody (1:100, ab9535, Abcam, Cambridge, UK), anti-IL-1 $\beta$ antibody (1:100, SC-7884, Santa Cruz Biotechnology, CA, USA), and anti-IL-6 antibody (1:100, SC-1265-R, Santa Cruz, CA, USA) overnight at $4{ }^{\circ} \mathrm{C}$. After incubating goat anti-rabbit secondary antibody (Boster, Wuhan, China), the samples were visualized with a 3,3-diaminobenzidine (DAB) kit (Boster, Wuhan, China). The mounted sections were observed and photographed under a microscope at 200x magnification (DM2500, Leica, Solms, Germany).

\section{Oxidative stress assessment}

Skin tissue homogenate from the burn wounds was prepared for direct or indirect detection of oxidative stress. ROS generation was detected by a DCFH-DA-based Reactive Oxygen Species Assay Kit (KGT010-1, KeyGEN Biotech, Nanjing, China), expressing as $n$-fold compared to that of the sham group. The indirect index of oxidative stress--the malondialdehyde (MDA) level was measured with a thiobarbituric acid reactive species (TBARS) assay kit (KGT003-1, KeyGEN Biotech, Nanjing, China), which were expressed in $\mathrm{nmol} / \mathrm{mg}$ protein. Tissue superoxide dismutase (SOD) activity was evaluated in the skin tissues of burn wounds and were measured using commercial assay kits from KeyGEN Biotech (KGT00150, Nanjing, China) according to the manufacturer's protocol. The results were expressed in $\mathrm{U} / \mathrm{mg}$ protein. Absorbance values were measured using a microplate reader (Model 680 Microplate Reader, BIO-RAD, CA, USA).

\section{Quantitative real time polymerase chain reaction (qRT-PCR) analysis}

In order to assess the release of inflammatory mediators further, qRT-PCR was introduced to determine the mRNA expression levels of myeloperoxidase (MPO), Interlaukin (IL)- $1 \beta$, and IL-6. Briefly, total RNA of frozen kidneys was extracted from tissues with TRIzol Reagent (Invit- rogen, Carlsbad, CA, USA) and RNase-Free DNase I (Qiagen, Duesseldorf, Germany). The SuperScript First- Strand Synthesis System for reverse transcription PCR (RT-PCR) (Invitrogen, Carlsbad, CA, USA) was applied to synthesise cDNAs, and RNA and CDNA concentrations and purities were measured via BIO-RAD spectrophotometry (SmartSpecTM Plus, BIO-RAD, CA, USA). The primers (Table 1) were designed using Primer Premier 6.0 software and were synthesised by Shanghai Biological Engineering Co., Ltd. (Shanghai, China). PCR amplifications were conducted using the Power SYBR ${ }^{\circledR}$ Master Mix (Invitrogen, Carlsbad, CA, USA) in an iQTM 5 Real-time PCR system (BIO-RAD, CA, USA). Expression levels were assessed relative to that of bactin, as an internal standard. Relative quantification of the target gene expression levels was conducted using the $2^{-\Delta \Delta C t}$ method.

\section{Western blot analysis}


Generally, the lysed protein samples were subjected to SDS-PAGE and transferred onto nitrocellulose membranes by electrophoresis, while aliquots of samples were used to determine the protein concentration of each sample using a bicinchoninic acid (BCA) kit (KGPBCA, KeyGEN Biotech, Nanjing, China). Subsequently, membranes were incubated in blocking buffer for $2 \mathrm{~h}$ and incubated overnight at 4 ${ }^{\circ} \mathrm{C}$ with the following primary antibodies: anti-TLR4 (1:200, SC-10741, Santa Cruz, CA, USA), anti-p-IKKa+b (1:500, ab2064, Abcam, Cambrige, UK), anti-IKKa+b (1:1000, ab178870, Abcam, Cambrige, UK), anti-pIKBa (1:1000, ab12135, Abcam, Cambrige, UK), anti-IKBa (1:500, ab32518, Abcam, Cambrige, UK), anti-pNF-kB p65(1:500, \#3033, Cell signaling Technology, Boston, USA), anti-NF-KB p65 (1:1000, ab16502, Abcam, Cambrige, UK), and anti-HO-1 (1:2000, ab13243, Abcam, Cambrige, UK). GAPDH (1:1500, ab8245, Abcam, Cambrige, UK) was used as a control on the same membranes. Applied secondary antibodies, the bands were detected with West Dura Extended Duration Substrate (Pierce, USA) and x-ray film (Kodak, USA) and then analyzed by Bandscan 5.0 software via comparison with GAPDH.

\section{Statistical analysis}

The data are presented as the means \pm standard deviation (SD). GraphPad Prism version 7 (San Diego, CA, USA) and SPSS 19 (SPSS, Chicago, IL, USA) were used for the statistical analysis. Multiple comparisons were analyzed with one-way analysis of variance (ANOVA) followed by a Bonferroni posthoc test. A value of $p<0.05$ was accepted as statistically significant.

\section{Results}

\section{Severe burn induced acute kidney injury in the early stage after injury, accompanying with increased oxidative stress and renal inflammation, whereas TAK242 or PDTC attenuates most of the above changes}

Similar to previous report, hematoxylin and eosin (HE) stained slices showed typical histological changes in kidney after burn injury under microscope, mainly presenting the absence of the proximal tubular brush border, blebbing of apical membranes, separation of tubular epithelial cells from the basement membrane, or aggregation of cells and proteins in the luminal region (Fig. 1a). Interestingly, different from vehicle1, the application of both selective TLR4 inhibitor-TAK242 and NF-KB activation inhibitor-PDTC seemed to be able to attenuate burn-induced histological damages in kidneys (Fig. 1a). Further, the tubular damage scores also showed a gradual and significant increase since $6 \mathrm{~h}$ till $48 \mathrm{~h}$ post burn (Fig. 1b). The scores of TAK242 and PDTC groups were significantly lower than that of $48 \mathrm{~h}$ group after burn, while there was no difference between those of vehicle1 and burn group at $48 \mathrm{~h}$ post burn (Fig. $1 \mathrm{~b}$ ).

In term of renal function, similarly we observed burn increased levels of sCr and NGAL since $6 \mathrm{~h}$ post burn and peaked at $48 \mathrm{~h}$ after burn, whereas TAK242 introduced a stronger effect to attenuate the burninduced increase of sCr and NGAL compared to PDTC. Vehicle1 acted no effect on the levels of $\mathrm{sCr}$ and NGAL in rats at $48 \mathrm{~h}$ after burn (Fig. 1c). 
In terms of oxidative status and secondary inflammation, we observed obvious ROS burst in rats' kidneys since the early stage $(6 \mathrm{~h})$ after burn, and persisted till $48 \mathrm{~h}$ post burn. Similarly, renal MDA level exhibited a significant increase from $6 \mathrm{~h}$ to $48 \mathrm{~h}$ after burn injury. Conversely, the representative endogenous antioxidant enzyme-SOD showed a remarked decrease after burn. The increased renal oxidative stress after burn conformed to the results of our previous study, nevertheless, none of vehicle1 and PDTC affected the burn-induced changes of ROS generation, and the levels of MDA and SOD. However, interestingly, TAK242 significantly decreased the burst of ROS and the MDA level in burned rats' kidneys $48 \mathrm{~h}$ after burn. Further, the renal mRNA levels of typical inflammatory mediators, as MPO, IL-1 $\beta$ and IL- 6 in burned rats increased significantly and gradually after insults, and peaked $48 \mathrm{~h}$ after burn, although there was a slight decrease happened $24 \mathrm{~h}$ post burn compared to the obvious elevation observed $6 \mathrm{~h}$ and $12 \mathrm{~h}$ post burn (Fig. 1e). Vehicle showed no effect on the mRNA transcription of MPO, IL-1 $\beta$ and IL-6, while both TAK242 and PDTC lowered the burn-induced increase of MPO, IL-1 $\beta$ and IL- 6 remarkably $48 \mathrm{~h}$ after burn insults (Fig. 1e).

\section{The activation TLR4/MyD88/NF-KB signaling pathway contributed to the regulation on the renal inflammation following burn injury}

We also investigated potential signal pathway related to renal inflammation induced by burn injury. It was observed typical green-positively labelled (FITC-labelled) renal tubules appeared since $12 \mathrm{~h}$ post burn, representing increased distribution of TLR4 (Fig. 2a). The increases continued to $48 \mathrm{~h}$ post burn (Fig. 2a). Different to the slight effect of vehicle, both TAK242 and PDTC were able to reduce the burn-induced increase of TLR4-postive tubules $48 \mathrm{~h}$ after insults, and the effect of TAK242 seemed to be more obvious. Further immunoblot detection supported the observation based on immunofluorescent staining, the expression of TLR4 showed a similar tendency (Fig. 2b). Specially, the TLR4 expression was significantly upregulated since $6 \mathrm{~h}$ post burn till $48 \mathrm{~h}$, while there was only a slight increase at $6 \mathrm{~h}$ after burn. TAK242 exhibited a stronger effect to reverse the remarked increase of TLR4 expression compared to that of PDTC. Vehicle acted no regulation on TLR4 expression. In view of the potential importance of MyD88 for TLR4 signaling pathway, we investigate the expression of MyD88 in rats' kidneys after burn (Fig. 2b). The results presented that the expression of MyD88 significantly increased since $6 \mathrm{~h}$ post burn, and showed a gradual tendency of increase with time like TLR4. TAK242 and PDTC obviously downregulated increased MyD88 expression $48 \mathrm{~h}$ post burn, while there is no differences between those of burn $48 \mathrm{~h}$ and vehicle groups.

A further immunoblot detection was taken to determine the possible activation of downstream signals following TLR4/MyD88 (Fig. 3). Finally, we observed a series of increased phosphorylation of inhibitor of nuclear factor kappa-B kinase subunit (IKK) $a+\beta$, NF-kappa-B inhibitor (IKB)a, NF-KB p65 after burn since $6 \mathrm{~h}$. All three peaks appeared at $48 \mathrm{~h}$. The inhibitory effect of TAK242 acted more significantly on the burn-induced phosphorylation of IKKa+ $\beta$ and $\mathrm{IKBa}$ in kidneys, whereas PDTC showed a more powerful effect to downregulate NF-KB p65 phosphorylation than TAK242. However, both of them were able to reverse the burn-induced phosphorylation of IKKa+ $\beta, \mathrm{IKBa}$, and NF-KB p65 at $48 \mathrm{~h}$ after insults, while vehicle showed no effect on this. 
All above indicated a typical activation of MyD88 dependent TLR4/NF-KB signaling pathway might be involved in the induction of early renal inflammation after burn injury.

\section{Atx Dose-relatively Attenuated Burn-induced Tissue Inflammation In Rats' Kidneys}

In our previous study, we had observed typical distribution of inflammatory mediators in burned rats' renal tissue based on the results of immuno-histological (IHC) staining. In this study, according to the results of IHC staining, we detected the increased MPO, IL-1 3 and IL- 6 immunoreactivity in the renal tubules of burned rats at the selected time window, similar to those of groups with a classic selective TLR4 activator-lipopolysaccharides (LPS) or an ATX solvent (vehicle) (Fig. 4). However, ATX seemed to be able to dose-relatedly decrease the number of MPO, IL-1 $\beta$ or IL-6 positive-stained tubular cells in rats $48 \mathrm{~h}$ after burn (Fig. 4). The results of IHC assessment were further supported by qRT-PCR detection (Fig. 4). The groups of burn $48 \mathrm{~h}$ and vehicle exhibited similar expressions of the different inflammatory mediators, like MPO, IL-1 $\beta$ and IL-6, although both showed a little weaker effect compared to those of LPS group. The levels of MPO, IL-1 $\beta$ and IL-6 in all three groups were significant higher than those of the Sham group. All dosages of ATX could downregulated burn-increased mRNA expression of MPO, IL-1 $\beta$ and IL- 6 at the time-point of $48 \mathrm{~h}$ after burn, and displayed a dose-related effect. The effect peaked at the dose of $20 \mathrm{mg} / \mathrm{kg}$. All these results suggested ATX might dose-relatively attenuated burn-induced inflammation in rats' kidneys.

\section{ATX downregulated the activation of TLR4/MyD88/NF-KB signaling pathway in kidneys after burn in a dose-related manner}

According to the aforementioned role of TLR4/MyD88/NF-KB signaling pathway on regulating burninduced renal tissue inflammation, we investigated the potential effect of ATX on the pathway (Fig. 5). The results of western blot showed that both LPS and burn insult could induced significantly elevation of TLR4, MyD88 and the secondary phosphorylation of IKKa+ $\beta, \mathrm{IKBa}$, and NF-KB p65, compared to those of the sham group. All three dosages of ATX were able to downregulate TLR4 and MyD88 expression remarkably, and the strongest effect appeared in the group of ATX $20 \mathrm{mg} / \mathrm{kg}$. In addition, a downstream downregulation of IKKa+ $\beta$, IKBa, and NF-KB p65 phosphorylation was also detected in all three ATX treatment groups. The peak of regulation was dose-related. Taken together, ATX seemed to be able to inhibit the activation of TLR4/MyD88/NF-KB signaling cascade.

\section{ATX ameliorated burn-induced renal inflammation through MyD88 dependent TLR4/NF-KB pathway}

Combined application of Selective TLR4 activator-LPS and TAK242 or PDTC was introduced to determine the anti-inflammation role of ATX based on TLR4/MyD88/NF-KB pathway in the progression of burn-induced AKI. Compared to the high dose of ATX, simultaneous LPS injection could remarkably abolish the downregulation of TLR4, MyD88 expression and phosphorylation of IKKa+ $\beta$, IKBa, and NF-KB p65 caused by ATX treatment in kidneys of burned rats (Fig. 6a). On the other hand, pretreatment of 
TAK242 could assist ATX to maintain the downregulation of the expression of TLR4, MyD88, phosphorylated IKKa+ $\beta$, phosphorylated IKBa, and phosphorylated NF-KB p65, whereas PDTC pretreatment showed a similar but weaker effect on this signaling pathway expect the phosphorylation of NF-kB p65. Furthermore, we observed an obvious increase of mRNA expression of selected inflammatory mediators (MPO, IL-1 $\beta$ and IL-6) in burned rats' kidneys with a combined application of high-dose ATX and LPS, compared to the single application of ATX (Fig. 6b). Both TAK 242 and PDTC could reverse the antagonism of LPS to the inhibitory effect of high-dose ATX on burn-induced inflammatory mediators release (Fig. 6b). Different from the results of western blot referring to TLR4 signaling cascade, PDTC exhibited a more powerful effect than TAK242 to recover the regulatory ability of ATX on inflammatory mediators. All of these supported the intermediate role of MyD88 dependent TLR4/NF-kB pathway in mediating the anti-inflammatory effect of ATX.

\section{ATX dose-related increased the distribution and expression of HO-1 in rats' kidneys after burn injury}

Based on the detection of Immunofluorescence staining, we observed increased red-labelled renal tubules $48 \mathrm{~h}$ after burn, representing HO-1 distribution. The administration of medium- and high-dose ATX in burned rats resulted in more red-labelled renal tubules than that of the burn group in the selected visual fields, while low-dose ATX showed a weaker ability to increase HO-1 distribution further (Fig. 7a). The vehicle solution of ATX showed no effect to affect burn-induced HO-1 expression (Fig. 7a). Furthermore, the immunoblot results exhibited burn insults induced a significant upregulation of HO-1 expression in kidneys of burned rats compared to that of the sham group, while medium and high dose ATX administration further increased the expression of renal HO-1 after burn (Fig. 7b). Both Vehicle and low dose ATX showed a slight effect to upregulate HO-1 expression compared to that of the burn group $48 \mathrm{~h}$ after injury (Fig. 7b). Collectively, ATX seemed to be able to further upregulate the protein level of renal HO-1 in burned rats in a dose-related manner.

\section{Discussion}

Severe burn insults not only bring about a large-scale trauma on skin, and it is also paralleled with a series of early complications in remote organs contributed to the severity and prognosis of burned patients ${ }^{33,34}$. In the early stage after burn, the existence of shock stage introduced several pathophysiological changes in circulation and local organs including overproduction of free radicals, release of pro-inflammatory cytokines, cell death, etc. ${ }^{34}$. We had suggested the crucial role of ROS in the development of early AKI after severe burn, which is highly related to the secondary molecule-biological changes, like apoptosis, in renal tissues ${ }^{7,8}$. We had also previously demonstrated ATX administration is a potentially-effective way to attenuate burn-induced early AKI in rats, due to its negative regulation on

oxidative damage and following activation of mitochondria-related apoptotic pathway ${ }^{7}$. In the present study, we were the first to initiate a systematic investigation on the role of local inflammation and potentially related regulators in the progression of early AKI after burn, and the potential antiinflammation based protective effect of ATX is also involved as a further study following our previous work. Herein, our findings showed as below, 1) The burn-caused renal inflammation contributes to the 
development of early AKI, paralleled with increased oxidative stress; 2) TLR4/NF-KB pathway is involved in the regulation on renal inflammation after burn insults dependent of MyD88; 3) ATX dose-relatedly attenuates the activation of TLR4/MyD88/NF-KB cascade and the following release of inflammatory mediators; 4) ATX further upregulates the renal expression of HO-1 in a dose-related manner to assist its regulation on oxidative stress and TLR4 pathway.

Commonly, AKI associates with histological alternation in regional tissue of kidney, especially tubules, basement, etc. ${ }^{35,36}$. In the early stage after burn, the risk of shock accompanied with a reduction of generalized renal blood flow (RBF) causes a series of endothelial and tubular changes, such as endothelial cell swelling, disruption of endothelial monolayer, etc., as a result of activation of coagulation system and impaired vascular reactivity, which contributes to early tubular damage post burn ${ }^{1}$. In the current study, we observed typical regional-changes in kidneys of severe burn model with time, including tubular epithelial necrosis, tubular dilation. Moreover, the histological progression in kidneys accompanied with the deterioration of renal function due to the results of classic functional indices- $\mathrm{sCr}$ and NGAL ${ }^{37}$. The following investigation revealed burn induced remarkable increase of ROS generation and decrease of anti-oxidant enzymes, representing increased oxidative stress, since $6 \mathrm{~h}$ post burn, and persisted during the early stage after burn, similar to our previous report ${ }^{8}$. The release of inflammatory mediators in kidneys exhibited a time-related increase after burn, and showed a late peak comparing with that of oxidative stress, which suggested it as a result of continuous ROS burst. After burn injury, the leukocytes, endothelial cells and the renal tubular epithelium contributes to the generation of several proinflammatory cytokines, like MPO, TNF-a, IL-6, IL-1 $\beta$, etc. ${ }^{8}$. Increased release of pro-inflammatory mediators is able to generate the enhanced vasoconstriction, vessel occlusion and decreased number of micro-vessels, which results in edema of outer medulla, increased tubular injury, and possible tubulointerstitial fibrosis ${ }^{38}$. In previous study, we had observed some clues to build a connection between ROS-related oxidative stress and local inflammation in burned rats' kidneys, and NF-kB seemed act a potential regulator to mediate burn-induced renal inflammation ${ }^{8}$. Due to previous reports, TLR4/NF-KB signaling pathway has been regarded as a classic signaling cascade participates in the regulation on transcription of pro-inflammatory mediators ${ }^{39,40}$. Other researchers exposed of importance are TLR4 and NF-KB in regulating innate and adaptive immune response reacting to renal ischemic injury $5,38,41$ and the role of TLR4/NF-KB cascade were also reported to be in in ischemic injuries of other organs by regulating immune and subsequent inflammatory response $\mathrm{e}^{42,43}$. Interestingly, we observed that both TAK242 and PDTC seemed to be able to attenuate the histological and functional deterioration of rats' kidneys $48 \mathrm{~h}$ after burn, which provided a clue about potential value of TLR4/NF-KB cascade in burn induced AKI. As a special inhibitor to TLR4, the histologic impact of TAK242 indicated potential role of TLR4 in burn induced AKI, while NF-KB inhibitor-PDTC suggested a pivotal role of NF-KB. Furthermore, administration of either TAK242 or PDTC could markedly attenuate elevated release of four pro-inflammatory mediators in burn-stimulated kidneys, which suggested the possible effect of TLR4/NF-KB signaling pathway on regulation of inflammation included in the development of burn induced AKI. However, only TAK242 exhibited an effect to lower the increased level of ROS in kidneys and enhance the activities of anti- 
oxidant enzymes after burn, indicating a potential relation between TLR4 and ROS-mediated oxidative stress.

In terms of typical TLR4/NF-KB signaling cascade, activated TLR4 could induced downstream phosphorylation of IKKs through a MyD88 dependent or independent route, and further led to the disintegration of IKK complex ${ }^{44,45}$. Subsequently, phosphorylation of IKBa gives a favor to the degradation of $1 \mathrm{kBa}$ and the following dissociation of NF-kB p65 subunit from IkBa/NF-kB/Rel complex ${ }^{44,45}$. Dissociated p65 subunit is activated via phosphorylation and then trans-locates into nucleus, which finally mediates transcription of multiple genes ${ }^{44,45}$. Several in vivo results from other researchers illustrated that of many kinds agents displayed a nephroprotective effect on ischemiareperfusion induced AKI by regulating TLR4/NF-KB pathway-mediated inflammation in kidneys s $^{11,46-48}$. On the other hand, TLR4/NF-KB signaling pathway also has been reported to involved in the pathogenesis of ischemic injuries in other organs, such as brain, heart, and liver ${ }^{40,49,50}$. In this study, we further investigated the detailed role of TLR4/NF-KB signaling cascade in burned rats via immunofluorescence staining and western blot. The time-related increase of distribution and expression of TLR4 and MyD88 suggested a MyD88 dependent manner involved in regulating the activation NF-KB upstream. Subsequently, the renal tissue of rat showed a sequentially-increased phosphorylation of signals, including IKKa $+\beta$, IKBa, NF-KB p65, in parallel to the increased release of pro-inflammatory cytokines. Combined with the reverse of TAK242 and PDTC blockade on renal structure, function and proinflammatory mediators, our results indicated MyD88 dependent TLR4/NF-KB signaling pathway involved in the regulation of burn-caused inflammation and related injury in kidneys, and might be a pivotal target to prevent or treat burn induced early AKI.

Astaxanthin (ATX), as a natural and strong antioxidant, can be extracted from multiple crustaceans ${ }^{51}$. It has been considered a safe and hopeful therapeutic agent in several diseases, as well as a safe feed additive and dietary supplement ${ }^{24,52-54}$. Our last study had primarily demonstrated the protective value of ATX on burn-induced early AKI, and the mechanism of action was related to its effects of attenuating oxidative stress and secondary renal cell apoptosis ${ }^{7}$. Inflammation is also the other crucial result following circular or tissue ROS burst after surgery or severe trauma. ATX has been suggested an antiinflammatory role based on several in vivo or in vitro researches referring to neurologic injury/dysfunction/diseases, dermatitis, lung injury, diabetes related disorder, tumor development and so on ${ }^{55-64}$. In terms of burn injury, we had observed the beneficial effect of ATX to prevent the deterioration of burn-wound through ameliorating tissue inflammation in wounds ${ }^{65}$. Existed results of prior studies suggested the anti-inflammatory property of ATX could be attributed to the regulation on certain inflammation-related signaling molecules, like NF-KB, JAK/STATS, PI3K/Akt, MAPK, Erk, MSK, etc., all of which might lead to secondary release of inflammatory cytokines ${ }^{61,63,64,66-69}$. Especially, NF-KB aroused more attention from researchers due to its crucial role in regulating inflammatory cascade under diverse pathophysiological status of multiple organs ${ }^{44}$. Furthermore, based on in vivo and in vitro subarachnoid hemorrhage (SAH) model, Zhang et al. demonstrated that TLR4 played a crucial role to mediate neuroinflammation caused by insults from SAH, while MyD88 and NF-KB acted downstream, and the 
neuroprotection of ATX mainly relied on inhibiting the TLR4 signaling pathway and the following proinflammatory response ${ }^{55}$. In the current study, our observations supported the importance of TLR4/MyD88/NF-KB signal axis on regulating renal inflammation after burn, and ATX exhibited a doserelated effect to inhibit the activation of TLR4 pathway and secondary increased release of inflammatory mediators. The single or combined application of classic TLR4 agonist-LPS and signal inhibitor TAK242 or PDTC led to the reverse or recovery of anti-inflammatory effect of high-dose ATX, which further indicated ATX could ameliorate burn-caused renal inflammation via regulating TLR4/MyD88/NF-KB signaling pathway.

Similar to apoptosis, inflammation is another orientation following ROS burst caused by multiple reasons. Under trauma or stress, there is certain inner biological defense to protect against possible organ or tissue injuries. Kidney is able to call adaptive and protective mechanisms to limit harmful effect ${ }^{5}$. As a heat shock protein, $\mathrm{HO}-1$ can be induced rapidly to deal with both oxidative and cellular stress, presenting powerful antioxidant or anti-inflammatory characteristics ${ }^{13,70,71}$. Futhermore, the previous researchers observed that a quick induction of HO-1 expression could be initiated in kidneys of AKI model even since 3 to 6 hours after both insults of ischemia/reperfusion and nephrotoxin ${ }^{13,16}$. In the prior and current study, we also detected an early upregulation of HO-1 in the kidney of burned rats, suggesting its potential value to regulate burn-induced AKI. As it reported, TLR4 is closely related to HO-1 expression, whereas HO-

1 can negatively regulate TLR4 through influencing ROS generation or some signals ${ }^{72,73}$. The anti-oxidant and anti-inflammatory properties of ATX also relied on the activation of HO-174-77. In a streptozotocininduced diabetic rat model, Yeh et al. found that ATX administration could attenuate diabetes-related ocular nerve injury by upregulating $\mathrm{HO}-1$ to decrease oxidative stress and secondary inflammation, and NF-KB acted downstream following HO-1 ${ }^{58}$. The beneficial potential of ATX for air inflammation was also observed in vivo and in vitro models, which was closely related to the participation of $\mathrm{HO}^{-1}{ }^{78}$. In this study, burn led to the remarkable increase of $\mathrm{HO}-1$ in renal tissue as response to thermal insults, whereas medium and high dose ATX asked a further upregulation of HO-1. Considering the regulation of ATX on ROS and inflammation in kidneys after burn, the data obtained supported our proposed role of HO-1 to mediate the regulation of ATX on renal inflammation through regulate oxidative stress and TLR4 in the development of early AKI after burn.

\section{Conclusion}

In summary, the current study demonstrated the local inflammation plays an important role in the development of burned induced early AKI, and MyD88 dependent TLR4/NF-KB pathway is involved in the regulation of renal inflammation (Fig. 8). ATX exhibited an anti-inflammation-based protection against early $\mathrm{AKI}$ after burn, and the beneficial effect can be attributed to its relief on burn-caused oxidative stress and secondary inflammation through targeting TLR4/MyD88/NF-KB axis. Further, HO-1 acted as a mediator to link the anti-oxidant and anti-inflammation of ATX. Our current finding further verified the potential value of ATX as a hopeful and safe way to improve the therapeutic outcome of burned patients during the early stage. 


\section{Abbreviations}

AKI

Acute kidney injury

IHC

Immunochemistry

IKK

Inhibitor of nuclear factor kappa-B kinase

$\mathrm{IKBa}$

nuclear factor of kappa light polypeptide gene enhancer in B-cells inhibitor, alpha

IL

Interleukin

$\mathrm{IR}$

ischemia-reperfusion

IP

intraperitoneal injection

MyD88

Myeloid differentiation primary response gene 88

NGAL

Neutrophil gelatinaseassociated lipocalin

$\mathrm{NF}$

Nuclear factor

qRT-PCR

Quantitative real time polymerase chain reaction

$\mathrm{sCr}$

Serum creatinine

TLR

Toll like receptor

\section{Declarations}

\section{Ethics approval and consent to participate}

The present study was performed according to protocols approved by the Committee on Animal Care and Use of the Second Affiliated Hospital Zhejiang University School of Medicine (2016-144), and strictly followed the National Institutes of Health Guidelines for the Care and Use of Laboratory Animals.

\section{Consent for publication}

Not applicable. 
The datasets used and/or analyzed during the present study are available from the corresponding author on reasonable request.

\section{Funding}

This work was supported by the following grants: National Natural Science Foundation of China (NSFC) grants of 81671909 and 81901958 and Zhejiang Provincial National Natural Science Foundation of China Grant of LY18H150004, LY19H150004 and LY20H150010.

\section{Author Contributions}

Conceptualization, S.G, Q.F. and M.Y.; methodology, S.G. and C.H.; validation, S.G., M.Y. and L.Z.; formal analysis, L.G. and C.Y.; investigation, S.G., L.G. and M.Y.; resources, M.Y. and C.Y.; data curation, Q.F.; writing-original draft preparation, S.G., L.G. Q.F. and X.G. writing-review and editing, S.G., Q.F. and X.G.; visualization, S.G.; supervision, C.H.; project administration, S.G. and C.H.. All authors have read and agreed to the published version of the manuscript.

\section{Competing interests}

The authors declare no conflict of interest.

\section{Acknowledgements}

No applicable.

\section{References}

1. Colpaert K, Hoste EA. Acute kidney injury in burns: a story of volume and inflammation. Crit Care. 2008;12:192.

2. Clark A, et al. Acute kidney injury after burn. Burns. 2017;43:898-908.

3. Thalji SZ, Kothari AN, Kuo PC, Mosier MJ. Acute Kidney Injury in Burn Patients. Ann Surg. 2017;266:376-82.

4. Elks $\mathrm{CM}$, et al. A blueberry-enriched diet attenuates nephropathy in a rat model of hypertension via reduction in oxidative stress. PLoS ONE. 2011;6:e24028.

5. Zuk A, Bonventre JV. Acute Kidney Injury. Annu Rev Med. 2016;67:293-307.

6. Mosier MJ, et al. Early acute kidney injury predicts progressive renal dysfunction and higher mortality in severely burned adults. J Burn Care Res. 2010;31:83-92.

7. Guo SX, et al. Astaxanthin attenuates early acute kidney injury following severe burns in rats by ameliorating oxidative stress and mitochondrial-related apoptosis. Mar Drugs. 2015;13:2105-23.

8. Guo SX, et al. Effects of hydrogen-rich saline on early acute kidney injury in severely burned rats by suppressing oxidative stress induced apoptosis and inflammation. J Transl Med. 2015;13:183. 
9. He L, et al. Mangiferin attenuate sepsis-induced acute kidney injury via antioxidant and antiinflammatory effects. Am J Nephrol. 2014;40:441-50.

10. et al. Signaling through the interleukin-18 receptor a attenuates inflammation in cisplatin-induced acute kidney injury. 82, 892-902 (2012).

11. O'Neill S, et al. Heat shock protein 90 inhibition abrogates TLR4-mediated NF-KB activity and reduces renal ischemia-reperfusion injury. Sci Rep. 2015;5:12958.

12. Nair AR, Masson GS, Ebenezer PJ, Del Piero F, Francis J. Role of TLR4 in lipopolysaccharide-induced acute kidney injury_ Protection by blueberry. Free Radic Biol Med. 2014;71:16-25.

13. Nath KA. Heme oxygenase-1 and acute kidney injury. 23, 17-24 (2014).

14. Carchman EH, Rao J, Loughran PA, Rosengart MR, Zuckerbraun BS. Heme oxygenase-1-mediated autophagy protects against hepatocyte cell death and hepatic injury from infection/sepsis in mice. Hepatology. 2011;53:2053-62.

15. Nath KA. Heme oxygenase-1: a provenance for cytoprotective pathways in the kidney and other tissues. Kidney Int. 2006;70:432-43.

16. Bolisetty S, et al. Heme Oxygenase-1 Inhibits Renal Tubular Macroautophagy in Acute Kidney Injury. J Am Soc Nephrol. 2010;21:1702-12.

17. Billings FT, Yu C, Byrne JG, Petracek MR, Pretorius M. Heme Oxygenase-1 and Acute Kidney Injury following Cardiac Surgery. Cardiorenal Med. 2014;4:12-21.

18. Gonçalves $\mathrm{GM}$, et al. The role of heme oxygenase 1 in rapamycin-induced renal dysfunction after ischemia and reperfusion injury. Kidney Int. 2006;70:1742-9.

19. Ambati R, Moi P, Ravi S, Aswathanarayana R, Astaxanthin. Sources, Extraction, Stability, Biological Activities and Its Commercial Applications-A Review. Mar Drugs. 2014;12:128-52.

20. Qiu X, et al. Protective effects of astaxanthin against ischemia/reperfusion induced renal injury in mice. J Transl Med. 2015;13:28.

21. Ghlissi Z, et al. Evaluation of efficacy of natural astaxanthin and vitamin $E$ in prevention of colistininduced nephrotoxicity in the rat model. 37, 960-966 (2014).

22. Kim YJ, Kim YA, Yokozawa T. Protection against oxidative stress, inflammation, and apoptosis of high-glucose-exposed proximal tubular epithelial cells by astaxanthin. J Agric Food Chem. 2009;57:8793-7.

23. Landon R, et al. Impact of Astaxanthin on Diabetes Pathogenesis and Chronic Complications. Mar Drugs. 2020;18:357-20.

24. Li J, Guo C, Wu J. Astaxanthin in Liver Health and Disease: A Potential Therapeutic Agent. Drug Des Devel Ther. 2020;14:2275-85.

25. Kidd P. Astaxanthin, cell membrane nutrient with diverse clinical benefits and anti-aging potential. Altern Med Rev. 2011;16:355-64.

26. Huang R-S, et al. Pharmacological Inhibition of Macrophage Toll-like Receptor 4/Nuclear Factorkappa B Alleviates Rhabdomyolysis-induced Acute Kidney Injury. Chin Med J. 2017;130:2163-9. 
27. Seki H, et al. Effect of Toll-like receptor 4 inhibitor on LPS-induced lung injury. Inflamm Res. 2010;59:837-45.

28. BS AEM, et al. Pyrrolidine dithiocarbamate improves mortality in a rat model of severe hemorrhage. $J$ Surg Res. 2013;179:e149-55.

29. Zhong X, et al. TAK242 suppresses the TLR4 signaling pathway and ameliorates DCD liver IRI in rats. Mol Med Rep. 2019;20:1-10.

30. Fang Q, et al. Astaxanthin protects against early burn-wound progression in rats by attenuating oxidative stress-induced inflammation and mitochondria-related apoptosis. Sci Rep. 2017;7:41440.

31. Plotnikov EY, et al. Mechanisms of LPS-Induced Acute Kidney Injury in Neonatal and Adult Rats. Antioxidants (Basel) 7, (2018).

32. Islam MS, Miao L, Yu H, Han Z, Sun H. Ethanol Extract of Illicium henryi Attenuates LPS-Induced Acute Kidney Injury in Mice via Regulating Inflammation and Oxidative Stress. Nutrients. 2019;11:1412-18.

33. Steinvall I, Bak Z, Sjoberg F. Acute kidney injury is common, parallels organ dysfunction or failure, and carries appreciable mortality in patients with major burns: a prospective exploratory cohort study. Crit Care. 2008;12:R124.

34. Parihar A, Parihar MS, Milner S, Bhat S. Oxidative stress and anti-oxidative mobilization in burn injury. Burns. 2008;34:6-17.

35. Feng $Y$, et al. Sustained oxidative stress causes late acute renal failure via duplex regulation on p38 MAPK and Akt phosphorylation in severely burned rats. PLoS ONE. 2013;8:e54593.

36. Sadek EM, Afifi NM, Elfattah LIA, Mohsen MA. A.-E. Histological study on effect of mesenchymal stem cell therapy on experimental renal injury induced by ischemia/reperfusion in male albino rat. Int J Stem Cells. 2013;6:55-66.

37. Ronco C. Acute kidney injury: from clinical to molecular diagnosis. Crit Care. 2016;20:201.

38. Bonventre JV, Yang L. Cellular pathophysiology of ischemic acute kidney injury. J Clin Invest. 2011;121:4210-21.

39. Chen $\mathrm{H}$, et al. Toll-like receptor 4 is involved in renoprotective effect of ischemic postconditioning after renal ischemia/reperfusion injury in rats. Urology. 2015;85:483.e1-7.

40. Chen H, Zhang R-Q, Wei X-G, Ren X-M, Gao X-Q. Mechanism of TLR-4/NF-KB pathway in myocardial ischemia reperfusion injury of mouse. Asian Pac J Trop Med. 2016;9:503-7.

41. Kanagasundaram NS. Pathophysiology of ischaemic acute kidney injury. Ann Clin Biochem. 2015;52:193-205.

42. Zhu L, et al. Exercise Preconditioning Regulates the Toll-Like Receptor 4/Nuclear Factor-kB Signaling Pathway and Reduces Cerebral Ischemia/Reperfusion Inflammatory Injury: A Study in Rats. J Stroke Cerebrovasc Dis. 2016. doi:10.1016/j.jstrokecerebrovasdis.2016.07.033.

43. $\mathrm{Li} \mathrm{J}$, et al. Resveratrol attenuates inflammation in the rat heart subjected to ischemia-reperfusion: Role of the TLR4/NF-KB signaling pathway. Mol Med Rep. 2015;11:1120-6. 
44. Hayden MS, Ghosh S. Shared principles in NF-kappaB signaling. Cell. 2008;132:344-62.

45. Campbell KJ, Perkins ND Regulation of NF-kappaB function. Biochem. Soc. Symp. 165-180 (2006).

46. Yang $Y$, et al. Renoprotective approaches and strategies in acute kidney injury. Pharmacol Ther. 2016;163:58-73.

47. Li Y-W, et al. Protective effect of tea polyphenols on renal ischemia/reperfusion injury via suppressing the activation of TLR4/NF-KB p65 signal pathway. Gene. 2014;542:46-51.

48. Wang $\mathrm{L}$, et al. Picroside II protects rat kidney against ischemia/reperfusion-induced oxidative stress and inflammation by the TLR4/NF-KB pathway. Experimental Therapeutic Medicine. 2015;9:1253-8.

49. Su X, Wang H, Zhao J, Pan H, Mao L. Beneficial Effects of Ethyl Pyruvate through Inhibiting HighMobility Group Box 1 Expression and TLR4/NF-B Pathway after Traumatic Brain Injury in the Rat. Mediators Inflamm. 2011;2011:1-10.

50. Tao X, et al. Total Flavonoids from Rosa laevigata Michx Fruit Ameliorates Hepatic Ischemia/Reperfusion Injury through Inhibition of Oxidative Stress and Inflammation in Rats. Nutrients. 2016;8:418.

51. Ambati RR, Phang SM, Ravi S, Aswathanarayana RG. Astaxanthin: sources, extraction, stability, biological activities and its commercial applications-a review. Mar Drugs. 2014;12:128-52.

52. Higuera-Ciapara I, Félix-Valenzuela L, Goycoolea FM, Astaxanthin. A Review of its Chemistry and Applications. Crit Rev Food Sci Nutr. 2006;46:185-96.

53. Valenti MT, Perduca M, Romanelli MG, Mottes M. \& Dalle Carbonare, L. A potential role for astaxanthin in the treatment of bone diseases (Review). Mol Med Rep. 2020;22:1695-701.

54. Brotosudarmo THP, Limantara L, Setiyono E. \& Heriyanto. Structures of Astaxanthin and Their Consequences for Therapeutic Application. Int J Food Sci. 2020;2020:2156582-16.

55. Zhang $X$, et al. Astaxanthin mitigates subarachnoid hemorrhage injury primarily by increasing sirtuin 1 and inhibiting the Toll-like receptor 4 signaling pathway. FASEB J. 2019;33:722-37.

56. Kumar A, Dhaliwal N, Dhaliwal J, Dharavath RN, Chopra K. Astaxanthin attenuates oxidative stress and inflammatory responses in complete Freund-adjuvant-induced arthritis in rats. Pharmacol Rep. 2020;72:104-14.

57. Bi J, Cui R, Li Z, Liu C, Zhang J. Astaxanthin alleviated acute lung injury by inhibiting oxidative/nitrative stress and the inflammatory response in mice. Biomed Pharmacother. 2017;95:974-82.

58. Yeh P-T, Huang H-W, Yang C-M, Yang W-S, Yang C-H. Astaxanthin Inhibits Expression of Retinal Oxidative Stress and Inflammatory Mediators in Streptozotocin-Induced Diabetic Rats. PLoS ONE. 2016;11:e0146438.

59. Ying C-J, et al. Anti-inflammatory Effect of Astaxanthin on the Sickness Behavior Induced by Diabetes Mellitus. Cell Mol Neurobiol. 2015;35:1027-37.

60. Park JH, et al. Anti-inflammatory effect of astaxanthin in phthalic anhydride-induced atopic dermatitis animal model. Exp Dermatol. 2018;27:378-85. 
61. Cai X, et al. Astaxanthin prevents against lipopolysaccharide-induced acute lung injury and sepsis via inhibiting activation of MAPK/NF-KB. Am J TransI Res. 2019;11:1884-94.

62. Cui $\mathrm{L}$, et al. Dietary natural astaxanthin at an early stage inhibits $\mathrm{N}$-nitrosomethylbenzylamineinduced esophageal cancer oxidative stress and inflammation via downregulation of NFKB and COX2 in F344 rats. Onco Targets Ther. 2019;12:5087-96.

63. Sun S-Q, Zhao Y-X, Li S-Y, Qiang J-W, Ji Y-Z. Anti-Tumor Effects of Astaxanthin by Inhibition of the Expression of STAT3 in Prostate Cancer. Mar Drugs. 2020;18:415.

64. Han J, et al. Astaxanthin Ameliorates Lipopolysaccharide-Induced Neuroinflammation, Oxidative Stress and Memory Dysfunction through Inactivation of the Signal Transducer and Activator of Transcription 3 Pathway. Mar Drugs. 2019;17:123-18.

65. Fang Q, et al. Astaxanthin protects against early burn-wound progression in rats by attenuating oxidative stress-induced inflammation and mitochondria-related apoptosis. Sci Rep. 2017;7:4144013.

66. Speranza L, et al. Astaxanthin Treatment Reduced Oxidative Induced Pro-Inflammatory Cytokines Secretion in U937: SHP-1 as a Novel Biological Target. Mar Drugs. 2012;10:890-9.

67. Fu J, et al. Astaxanthin alleviates spinal cord ischemia- reperfusion injury via activation of PI3K/ Akt/GSK-3ß pathway in rats. 1-11 (2020). doi:10.1186/s13018-020-01790-8.

68. Kim YH, Koh H-K, Kim D-S. Down-regulation of IL-6 production by astaxanthin via ERK-, MSK-, and NF-KB-mediated signals in activated microglia. Int Immunopharmacol. 2010;10:1560-72.

69. Zhang X-S, et al. Astaxanthin offers neuroprotection and reduces neuroinflammation in experimental subarachnoid hemorrhage. J Surg Res. 2014;192:206-13.

70. Bak S-U, et al. Heme oxygenase-1 (HO-1)/carbon monoxide (CO) axis suppresses RANKL-induced osteoclastic differentiation by inhibiting redox-sensitive NF-KB activation. BMB Rep. 2017;50:103-8.

71. Sudan $\mathrm{K}$, et al. TLR4 activation alters labile heme levels to regulate $\mathrm{BACH} 1$ and heme oxygenase-1 expression in macrophages. Free Radic Biol Med. 2019;137:131-42.

72. Mandal $P$, et al. Adiponectin and heme oxygenase-1 suppress TLR4/MyD88-independent signaling in rat Kupffer cells and in mice after chronic ethanol exposure. J Immunol. 2010;185:4928-37.

73. Chillappagari S, et al. Impaired TLR4 and HIF expression in cystic fibrosis bronchial epithelial cells downregulates hemeoxygenase-1 and alters iron homeostasis in vitro. Am J Physiol Lung Cell Mol Physiol. 2014;307:L791-9.

74. Wang $\mathrm{H}-\mathrm{Q}$, et al. Astaxanthin upregulates heme oxygenase-1 expression through ERK1/2 pathway and its protective effect against beta-amyloid-induced cytotoxicity in SH-SY5Y cells. Brain Res. 2010;1360:159-67.

75. Ye Q, Huang B, Zhang X, Zhu Y, Chen X. Astaxanthin protects against MPP(+)-induced oxidative stress in PC12 cells via the HO-1/NOX2 axis. BMC Neurosci. 2012;13:156.

76. Wang $Y$, et al. Remote Ischemic Preconditioning Protects against Liver Ischemia-Reperfusion Injury via Heme Oxygenase-1-Induced Autophagy. PLoS ONE. 2014;9:e98834-12. 
77. Wu H, et al. Astaxanthin as a Potential Neuroprotective Agent for Neurological Diseases. Mar Drugs. $2015 ; 13: 5750-66$.

78. Nian L, et al. Astaxanthin suppresses cigarette smoke and lipopolysaccharide-induced airway inflammation through induction of heme oxygenase-1. Cell Mol Biol (Noisy-le-grand). 2019;65:94-9.

\section{Tables}

Table 1 The oligonucleotide primers used for PCR amplification

\begin{tabular}{|c|c|c|c|c|}
\hline Gene & $\begin{array}{l}\text { Genbank } \\
\text { Accession }\end{array}$ & Primer Sequences (5'to3') & $\begin{array}{l}\text { Size } \\
\text { (bp) }\end{array}$ & $\begin{array}{l}\text { Annealing } \\
\left({ }^{\circ} \mathrm{C}\right)\end{array}$ \\
\hline \multirow[t]{2}{*}{ Rat MPO } & \multirow[t]{2}{*}{ NM_001107036.1 } & GCTACGGGATGGCGATAGGTTT & \multirow[t]{2}{*}{126} & \multirow[t]{2}{*}{63} \\
\hline & & GACACGGTAGTGATGCCAGTGTT & & \\
\hline \multirow[t]{2}{*}{ Rat IL-1b } & \multirow[t]{2}{*}{ NM_031512.2 } & CCTAGGAAACAGCAATGGTCGGGAC & \multirow[t]{2}{*}{126} & \multirow[t]{2}{*}{63} \\
\hline & & GTCAGAGGCAGGGAGGGAAACAC & & \\
\hline \multirow[t]{2}{*}{ Rat IL-6 } & \multirow[t]{2}{*}{ NM_012589.2 } & CAATCAGAATTGCCATTGCACAA & \multirow[t]{2}{*}{169} & \multirow[t]{2}{*}{63} \\
\hline & & CGTCTTCGCAAGAGGAAGAGCAGT & & \\
\hline $\begin{array}{l}\text { Rat b- } \\
\text { actin }\end{array}$ & EF156276.1 & CCAACCGTGAAAAGATGACCCAGAT & 97 & 63 \\
\hline
\end{tabular}

\section{Figures}


a

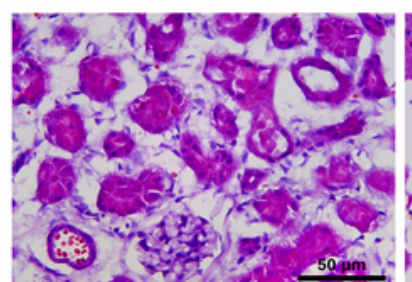

Sham

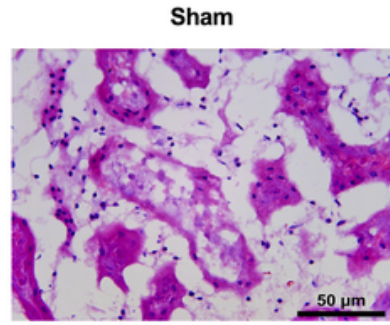

Burn 48 h

b

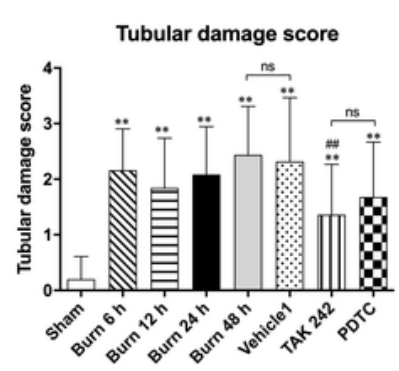

d
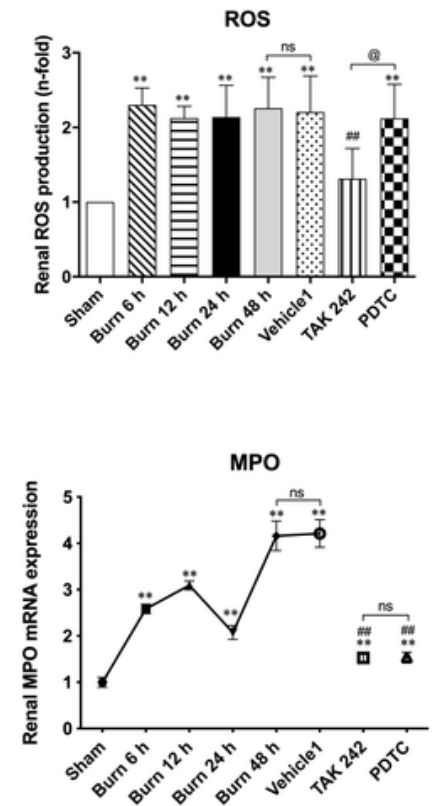

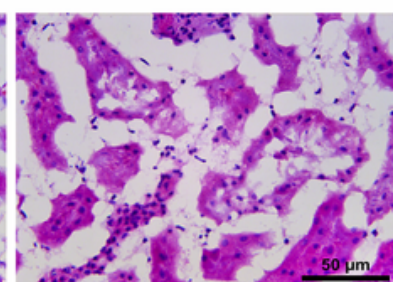

Burn $6 \mathrm{~h}$

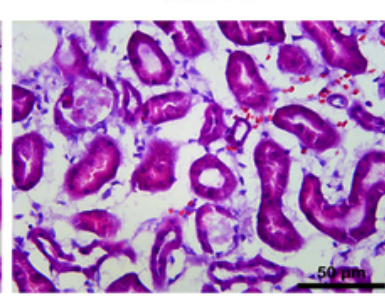

Vehicle

C
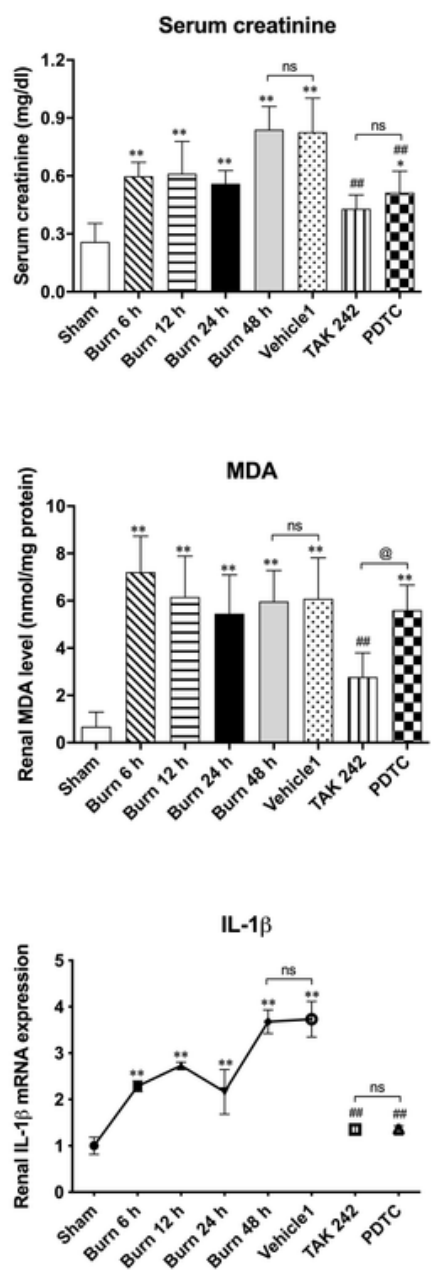

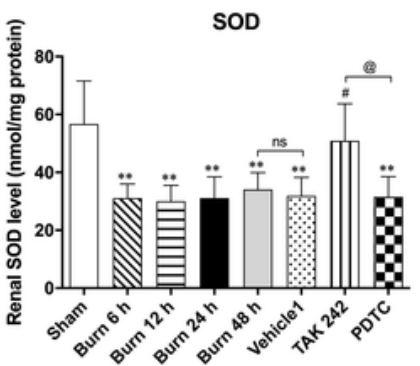

Burn $24 \mathrm{~h}$

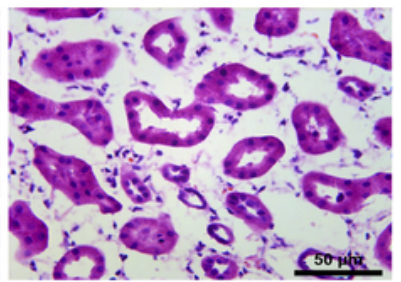

PDTC

Serum NGAL

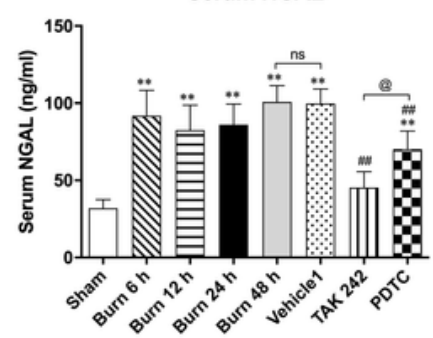

SOD

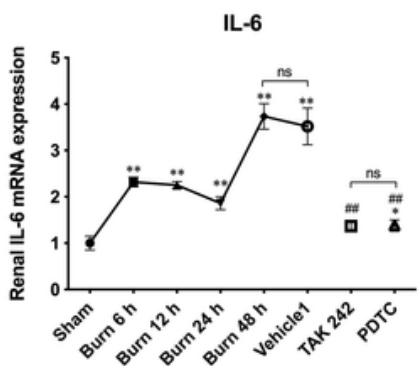

Figure 1

Histological, functional and biological assessments of kidney after burn. a Representative HE-stained images of renal tissues at a magnification of 200x; b Tubular damage score according to HE-stained slices; c Blood-based detection of creatinine and NGAL; d Oxidative stress evaluation based on commercial kits of ROS, MDA and SOD; e The release levels of inflammatory mediators after insults. The 
sample size was $n=8$ for each group. The results are expressed as the mean $\pm S D$. ${ }^{*} p<0.05, * \star p<0.01$, v.s. sham; \# p<0.05, \#\# p<0.01, v.s. vehicle1; @ p<0.05, ns p >0.05.।

a

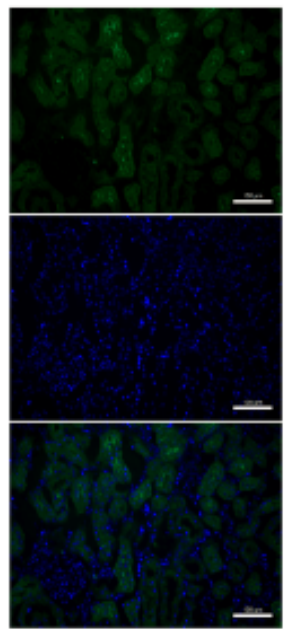

Sham

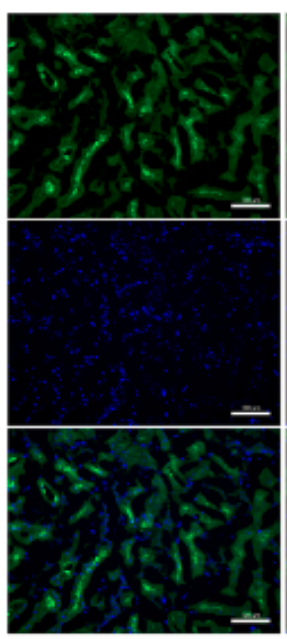

Burn $48 \mathrm{~h}$

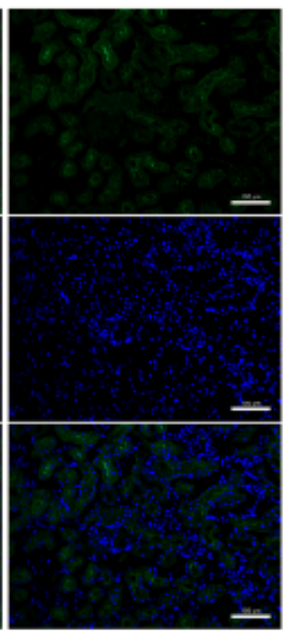

Burn $6 \mathrm{~h}$

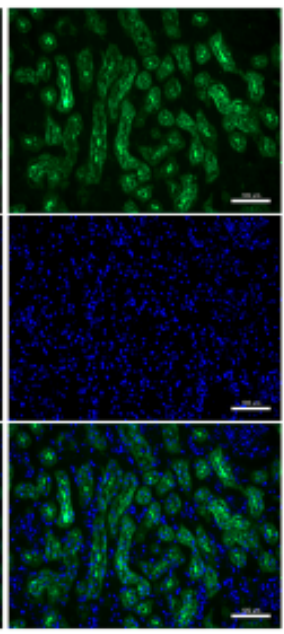

Vehicle1

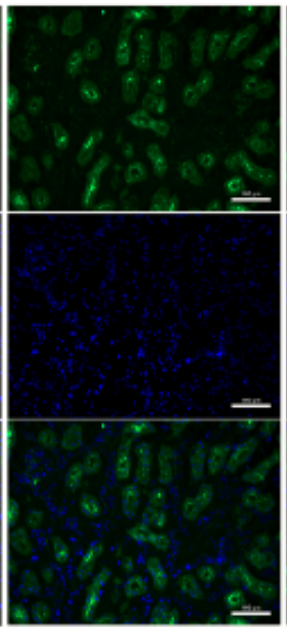

Burn $12 \mathrm{~h}$

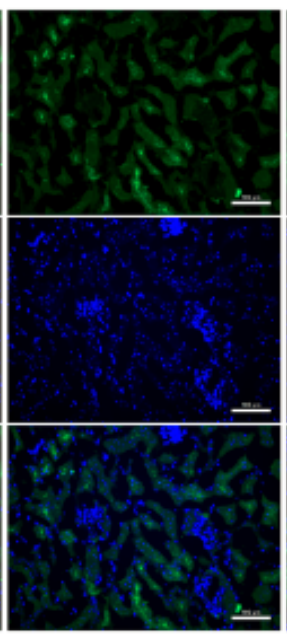

TAK 242

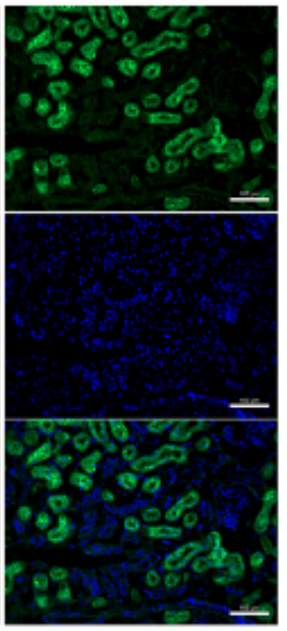

Bum $24 \mathrm{~h}$

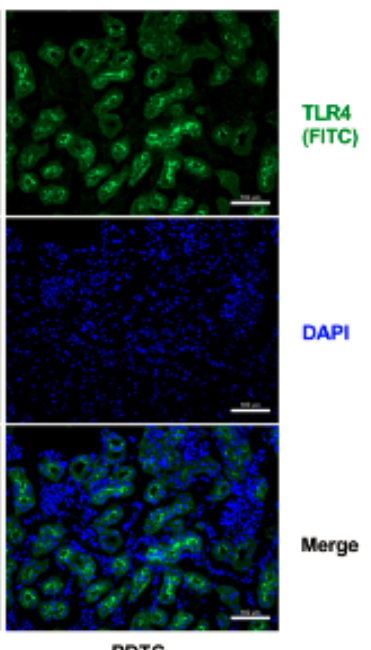

b

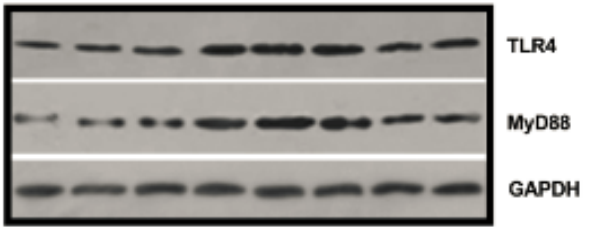

Sham $6 \mathrm{~h} \quad 12 \mathrm{~h} \quad 24 \mathrm{~h} \quad 48 \mathrm{~h}$ Vehicle1 TAK PDTC

242
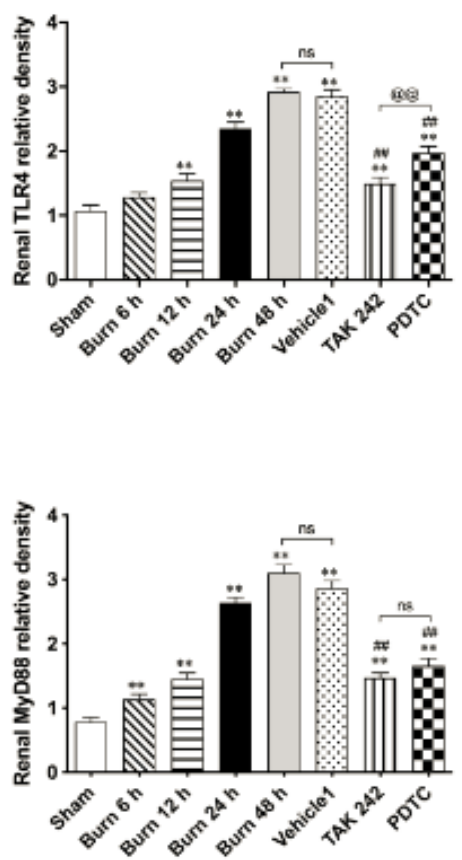

Figure 2

Immunofluorescent and immunoblotting detection of TLR4 pathway. a Immunofluorescence double staining of TLR4; $b$ Representative bands of Western blot on TLR4 and MyD88. The sample size was $n=$ 6 for each group. The results are expressed as the mean \pm SD. ${ }^{*} p<0.05,{ }^{\star *} p<0.01$, v.s. sham; \# $p<0.05$, \#\# p<0.01, v.s.vehicle1; @@ p<0.01,ns p >0.05. 


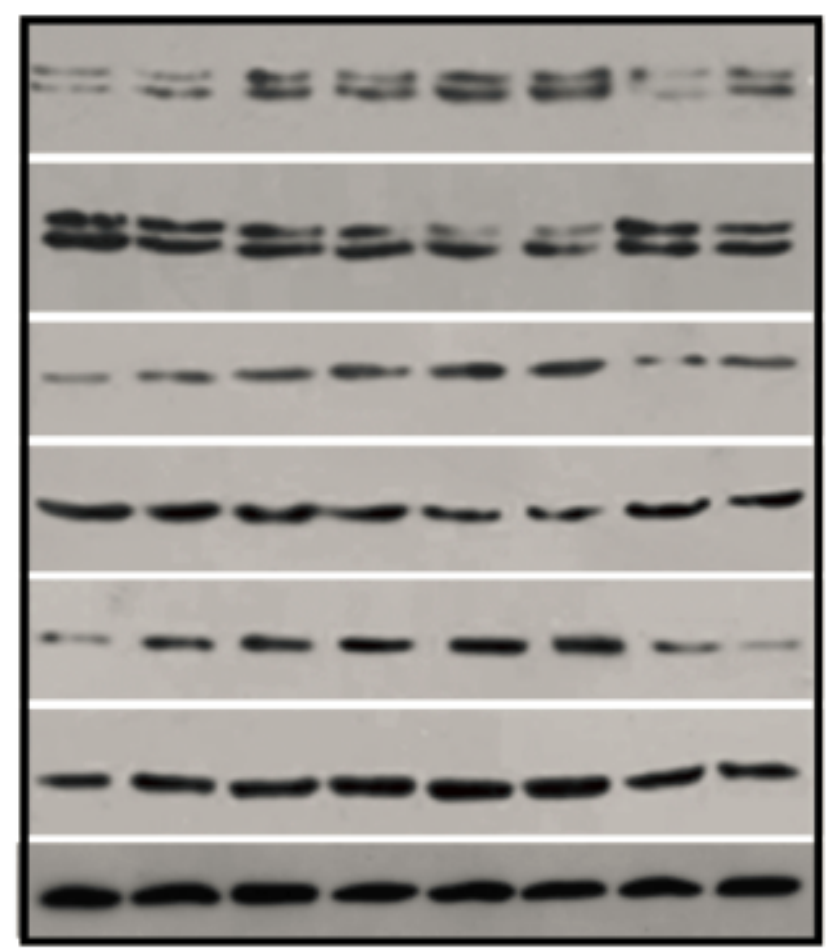

Sham $6 \mathrm{~h} \quad 12 \mathrm{~h} \quad 24 \mathrm{~h} \quad 48 \mathrm{~h}$ Vehicle1 TAK PDTC 242

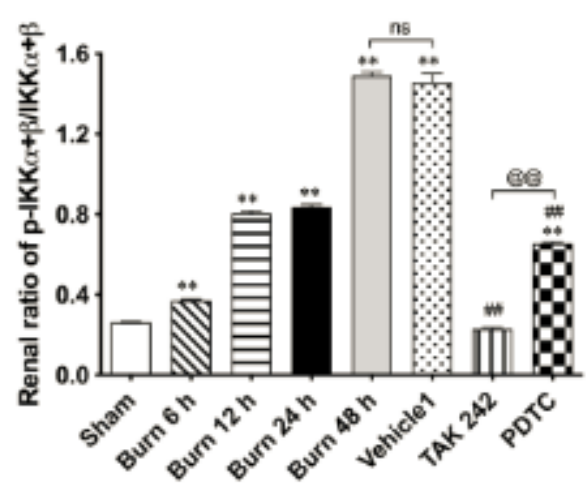

$\mathrm{p}-\mathrm{I} \mathrm{B} \alpha$

$\mathrm{I \kappa B} \alpha$

p-NF-kB p65

NF-кB p65
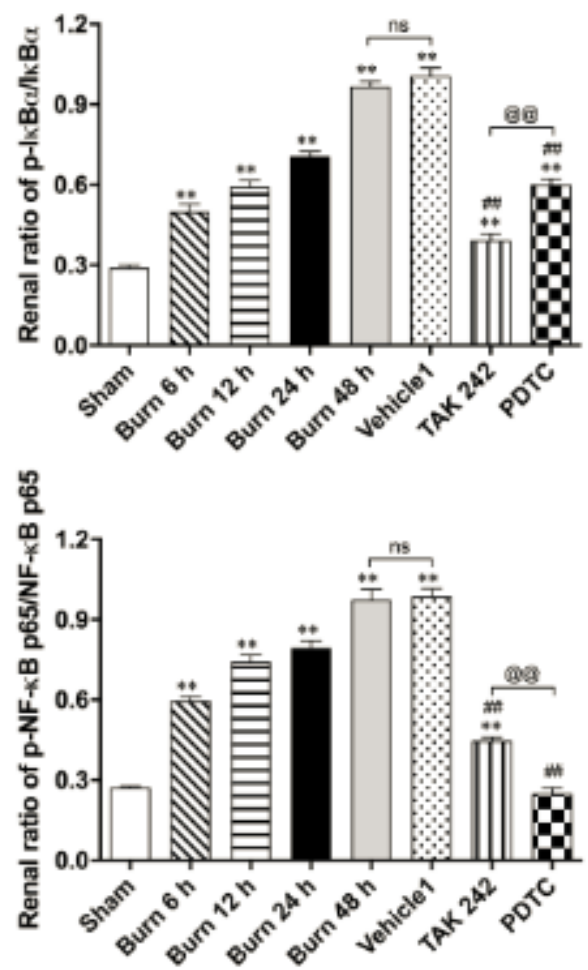

Figure 3

Immunoblotting detection on downstream signals of MyD88 dependent TLR4 pathway. The results are expressed as the mean $\pm S D$. * $p<0.05$, ${ }^{*} p<0.01$, v.s. sham; $\# p<0.05, \# \#<<0.01$, v.s. vehicle $1 ; @ p<0.05$, @@p<0.01,ns p >0.05. 


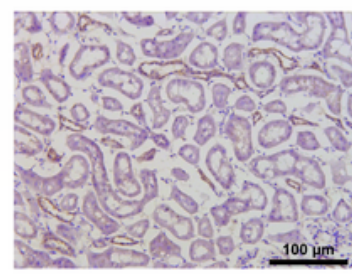

Sham

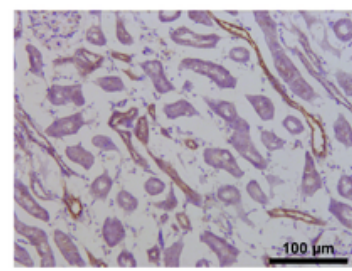

ATX $5 \mathrm{mg} / \mathrm{kg}$

b

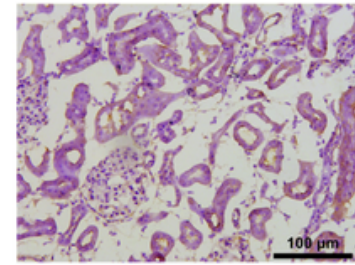

Sham

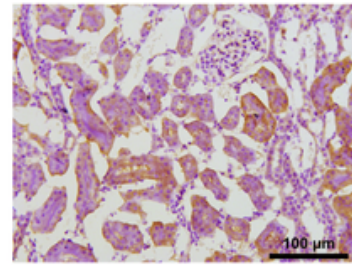

ATX $5 \mathrm{mg} / \mathrm{kg}$

C

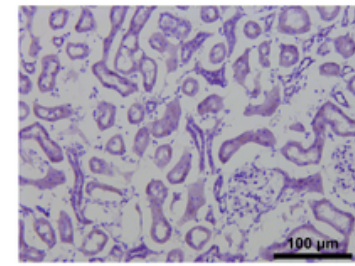

Sham

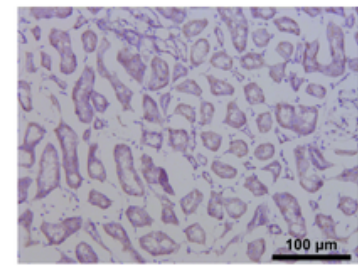

ATX $5 \mathrm{mg} / \mathrm{kg}$

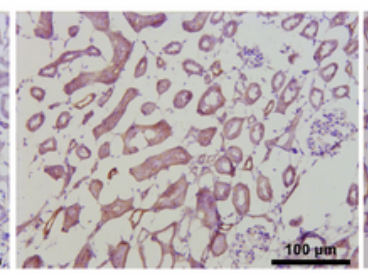

LPS

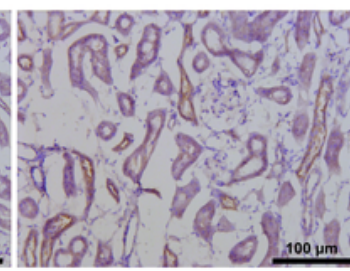

ATX $10 \mathrm{mg} / \mathrm{kg}$

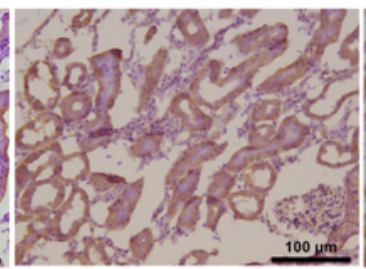

LPS

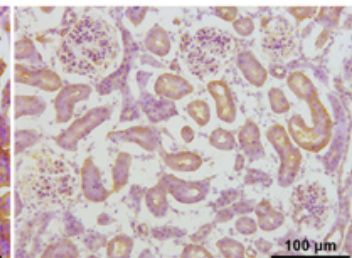

ATX $10 \mathrm{mg} / \mathrm{kg}$

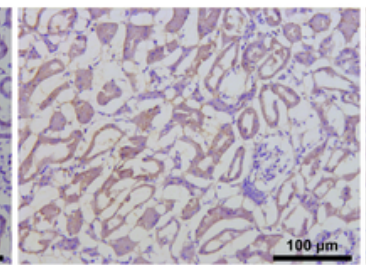

LPS

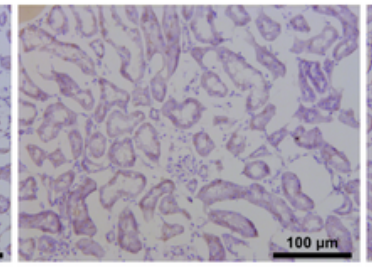

ATX $10 \mathrm{mg} / \mathrm{kg}$

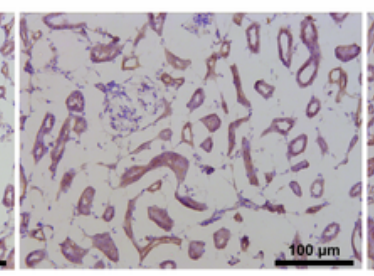

Burn $48 \mathrm{~h}$

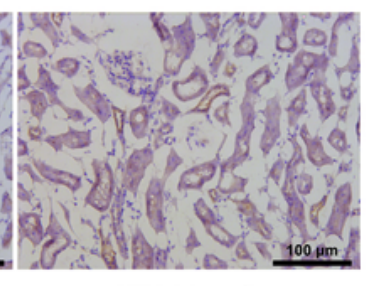

ATX $20 \mathrm{mg} / \mathrm{kg}$

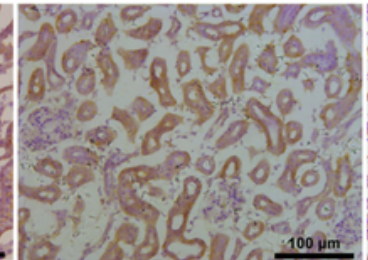

Burn 48 h

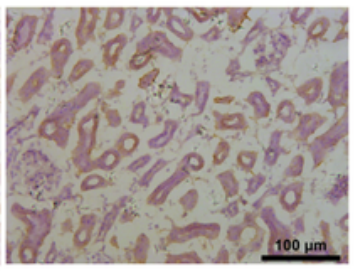

ATX $20 \mathrm{mg} / \mathrm{kg}$

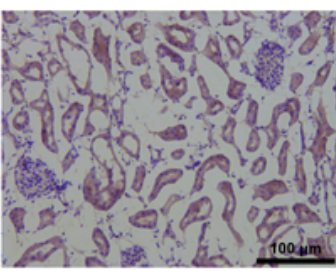

Burn 48 h

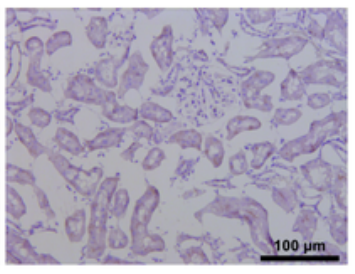

ATX $20 \mathrm{mg} / \mathrm{kg}$

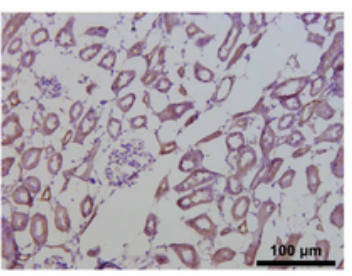

Vehicle2
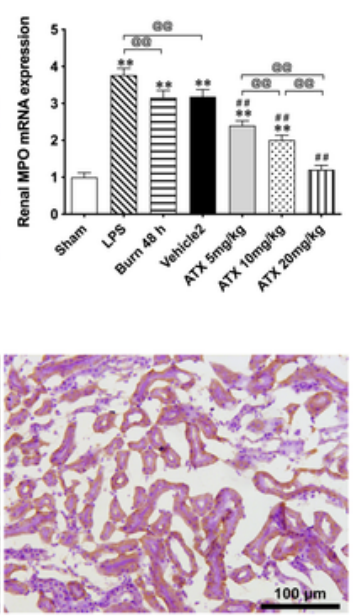

Vehicle2
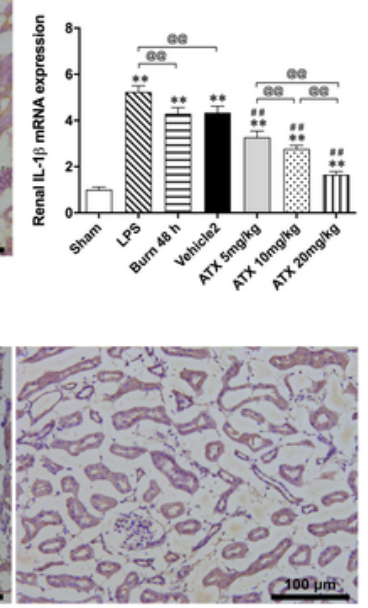

Vehicle2

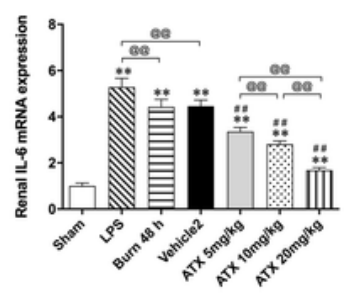

\section{Figure 4}

Astaxanthin dose-relatively decreases the burn-induced release of inflammatory mediators. a Representative immunohistochemical images and tissue mRNA levels of MPO with ATX treatment; $b$ Representative immunohistochemical images and tissue mRNA levels of IL-1区 with ATX treatment; c Representative immunohistochemical images and tissue mRNA levels of IL- 6 with ATX treatment. The 
results are expressed as the mean $\pm S D$. ${ }^{*} p<0.05$, ${ }^{\star *} p<0.01$, v.s. sham; $\# p<0.05, \# \# p<0.01$, v.s. vehicle2; @ $p<0.05$, @ $0<0.01, n s p>0.05$.

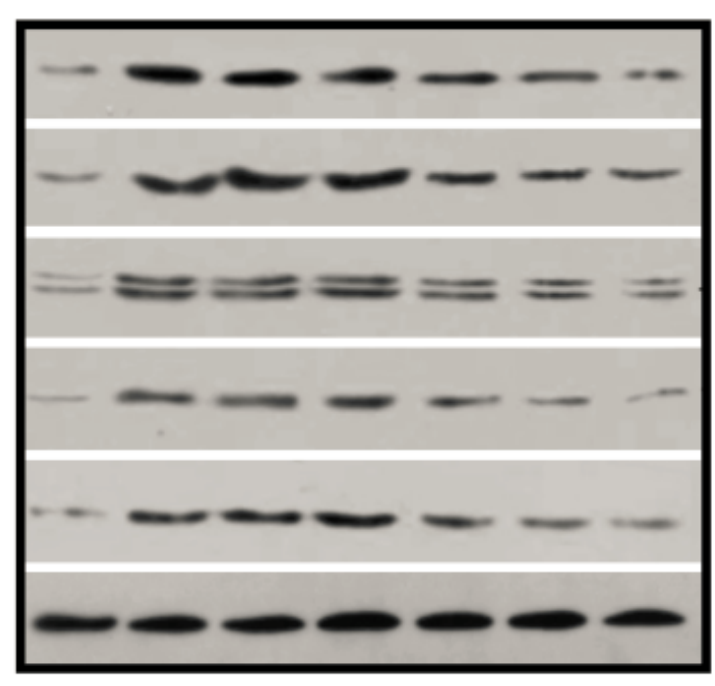

Sham LPS Burn Vehicle2 ATX ATX ATX

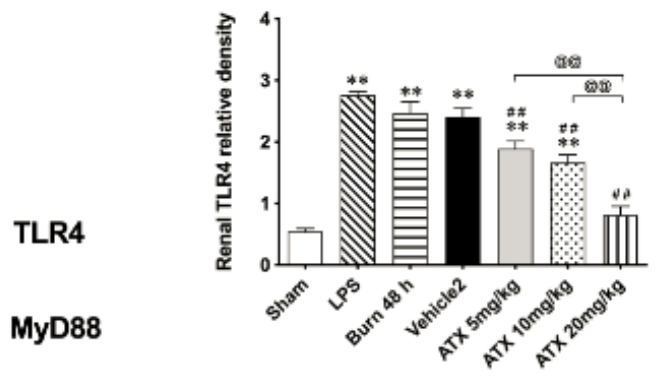

MyD88
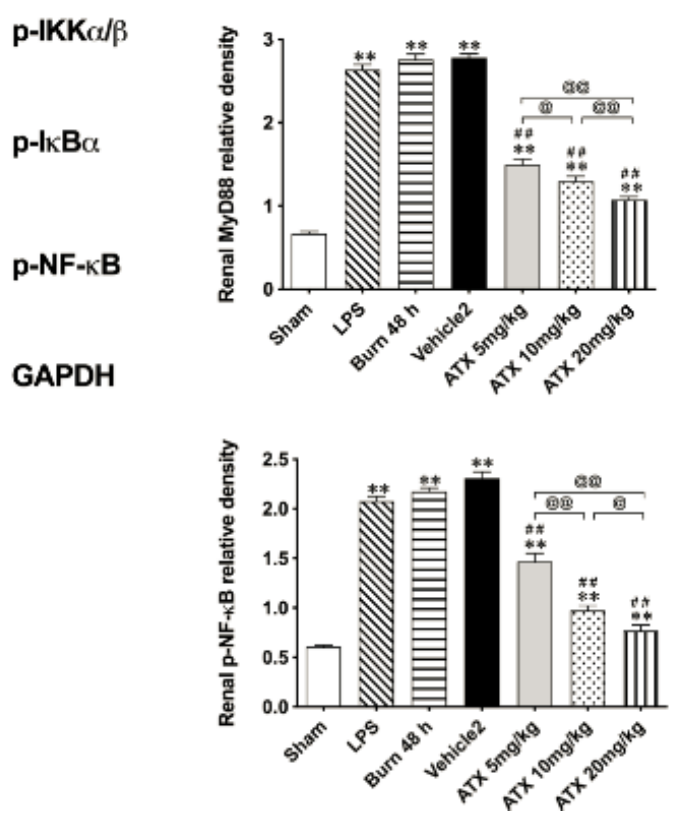
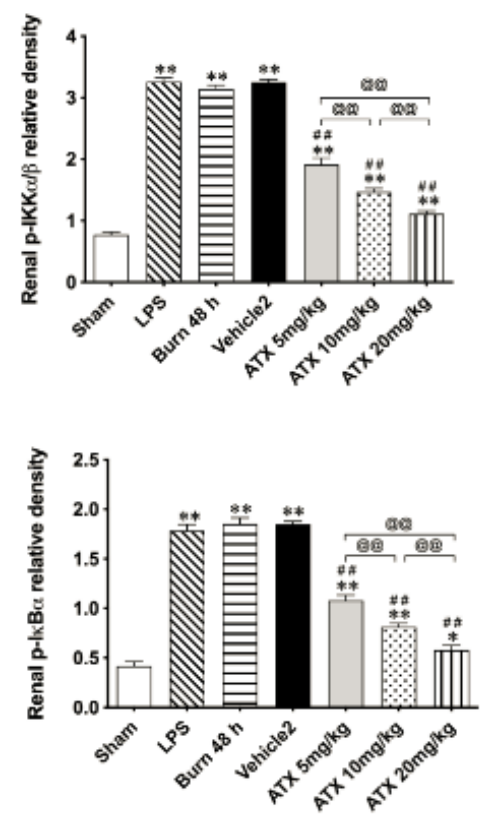

\section{Figure 5}

Astaxanthin dose-relatively downregulates the activation of TLR4/myD88/NF-nB signaling pathway. The results are expressed as the mean $\pm S D .{ }^{*} p<0.05$, ${ }^{* *} p<0.01$, v.s. sham; $\# p<0.05$, \#\# $p<0.01$, v.s. vehicle2; @ $p<0.05$, @ $0<0.01, n s p>0.05$. 

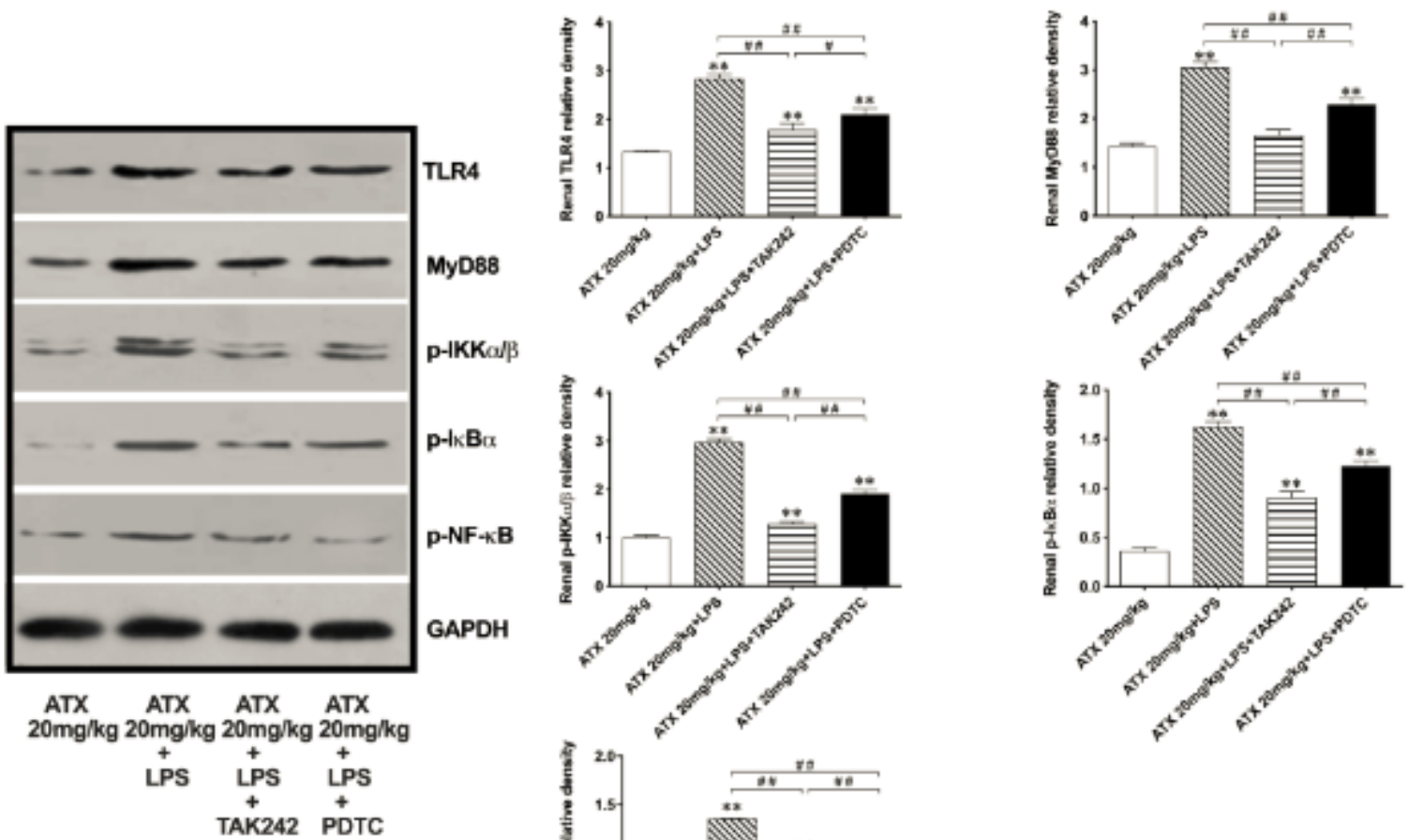

b
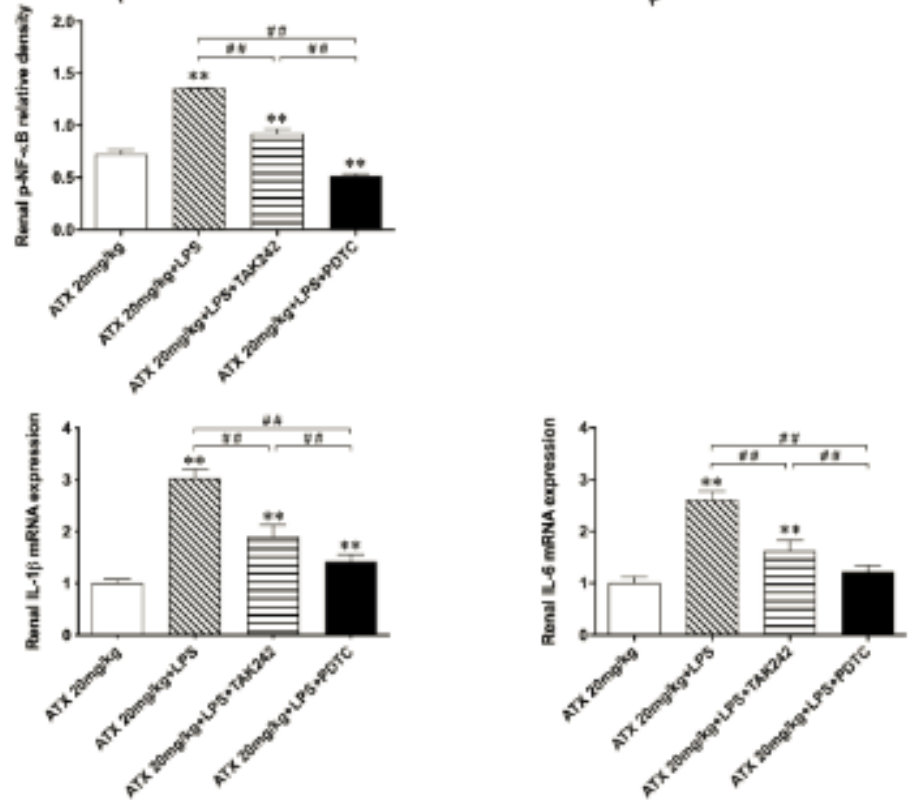

Figure 6

Astaxanthin attenuates burn-induced inflammation through regulating TLR4/MyD88/NF-AB pathway. a Representative bands of western blot are shown after introduction of LPS, TAK242 and PDTC. LPS reversed the effect of high-dose ATX on expression of TLR4, MyD88 and downstream phosphorylated activation of IKK区/区, IQ, BDNF- $\square B$, while both TAK242 and PDTC could help to recover the effect of ATX; $b$ The inhibition of ATX on release of selected inflammatory mediators could be abolished by LPS, and resumed by using TAK242 or PDTC. The results are expressed as the mean $\pm S D$. ${ }^{*} p<0.05,{ }^{\star} * p<0.01$, v.s. ATX $20 \mathrm{mg} / \mathrm{kg} ; \# \mathrm{p}<0.05, \# \# \mathrm{p}<0.01$. 
a

HO-1

DAPI
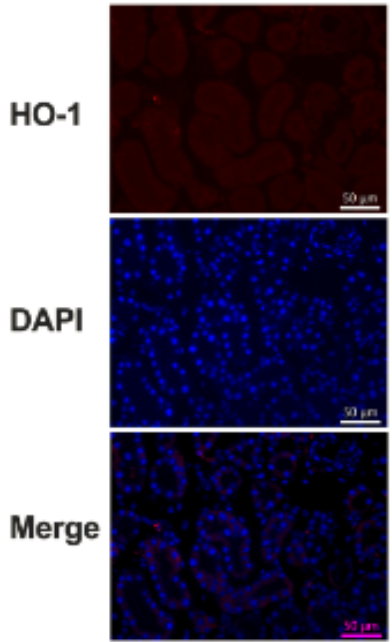

Sham

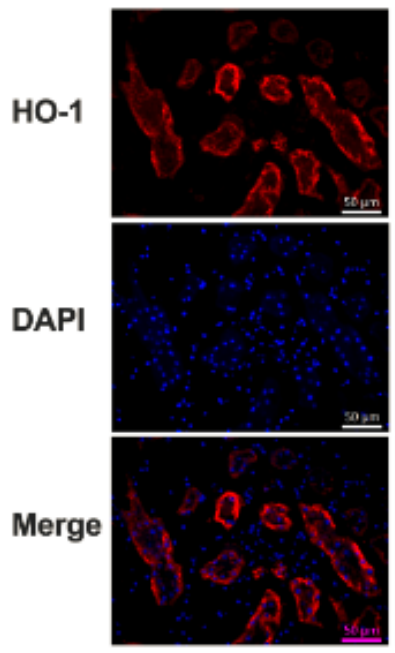

ATX $5 \mathrm{mg} / \mathrm{kg}$
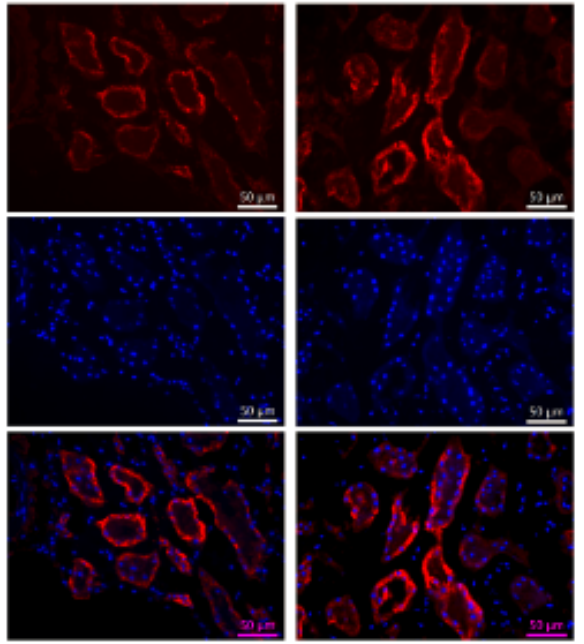

Burn
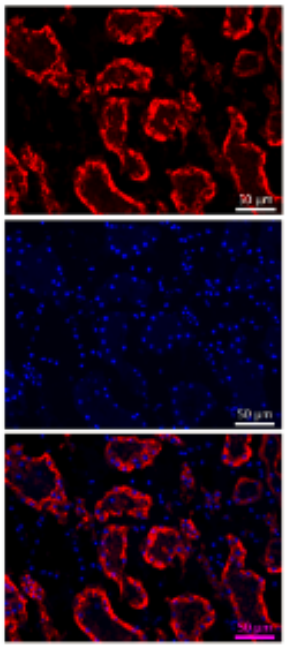

ATX $10 \mathrm{mg} / \mathrm{kg}$

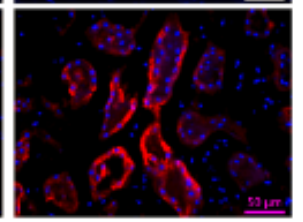

Vehicle2
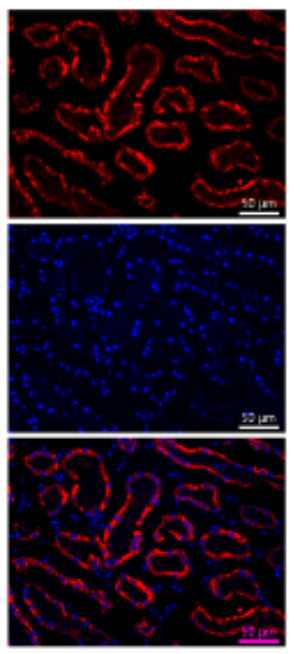

ATX 20mg/kg b

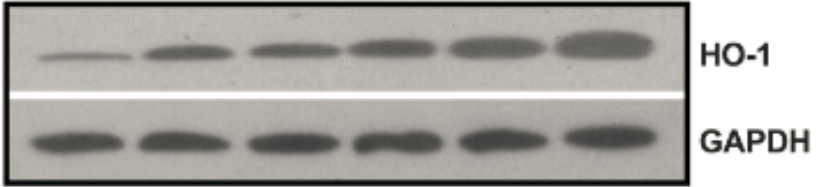

Sham Burn Vehicle2 ATX ATX ATX

$5 \quad 10 \quad 20$ $\mathrm{mg} / \mathrm{kg} \mathrm{mg} / \mathrm{kg} \mathrm{mg} / \mathrm{kg}$

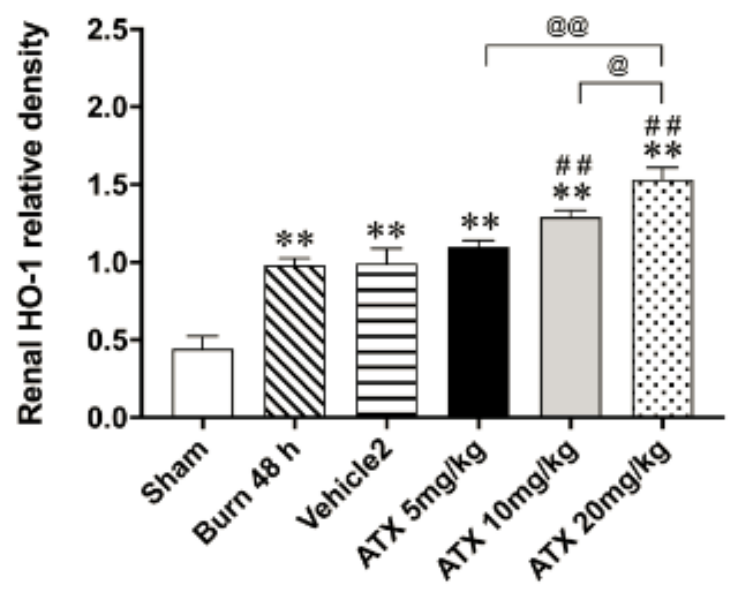

Figure 7

Astaxanthin treatment dose-related increased the distribution and expression of HO-1 in kidneys of burned rats. a Representative images of Immunofluorescence staining of HO-1 in Kidneys; b

Representative bands of HO-1 protein expression. The results are expressed as the mean $\pm S D$. * $p<0.05$, ** $p<0.01$, v.s. sham; \#\# p<0.01, v.s. vehicle2; @ p<0.05, @@ p<0.01, ns $p>0.05$. 


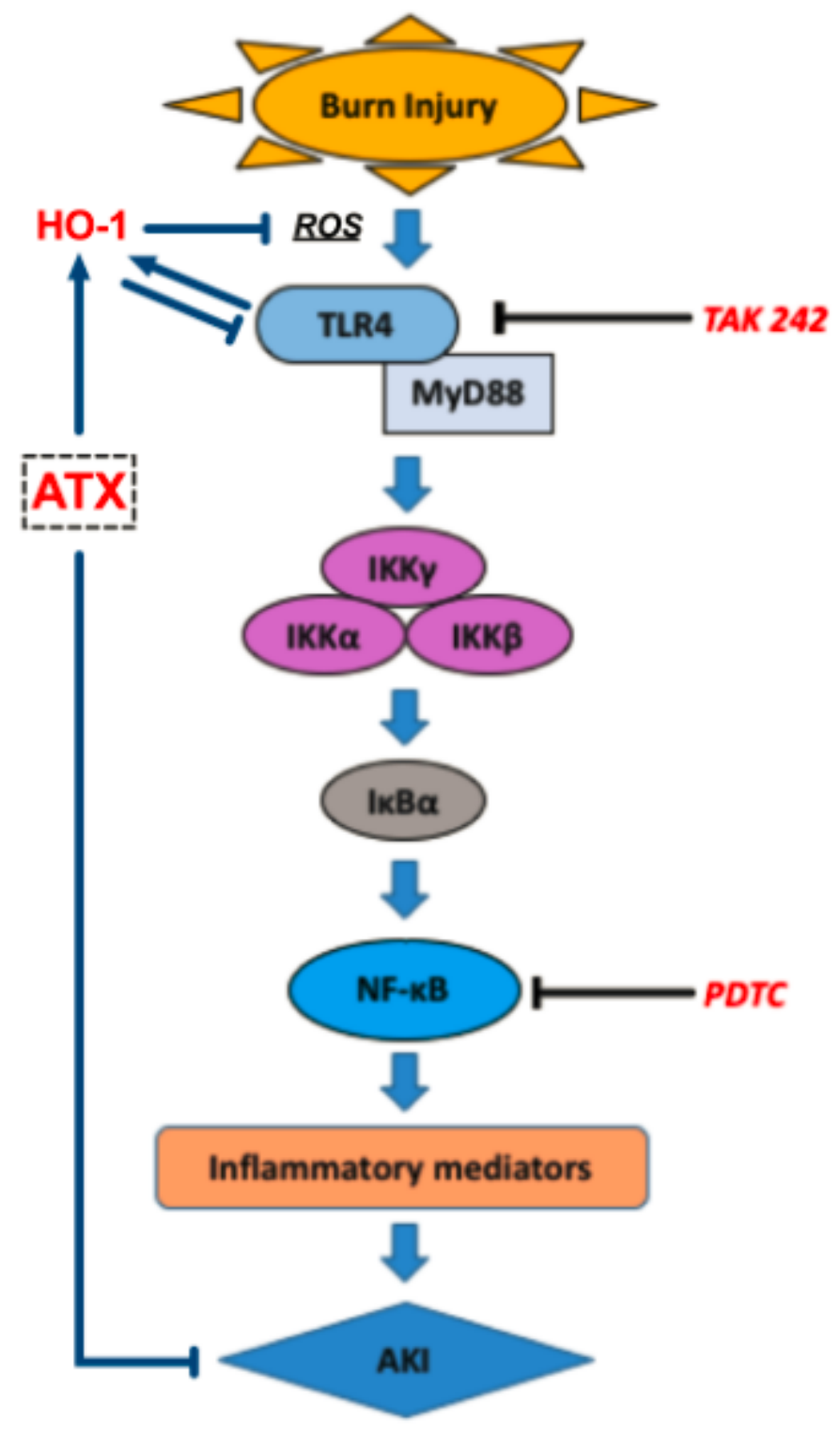

Figure 8

The schematic diagram of this study. 